\title{
Mobile Health Interventions for Physical Activity, Sedentary Behavior, and Sleep in Adults Aged 50 Years and Older: A Systematic Literature Review
}

\author{
Steriani Elavsky, Lenka Knapova, Adam Klocek, and David Smahel
}

\begin{abstract}
We provide a systematic review of interventions utilizing mobile technology to alter physical activity, sedentary behavior, and sleep among adults aged 50 years and older. A systematic search identified 52 relevant articles (randomized control trial [RCT], quasi-experimental, pre/post single-group design). Of 50 trials assessing physical activity, 17 out of 29 RCTs and 13 out of 21 trials assessed for pre/post changes only supported the effectiveness of mobile interventions to improve physical activity, and 9 studies (five out of 10 RCTs and all four pre/post studies) out of 14 reduced sedentary behavior. Only two of five interventions improved sleep (one out of two RCTs and one out of three pre/post studies). Text messaging was the most frequently used intervention (60\% of all studies) but was usually used in combination with other components (79\% of hybrid interventions included SMS, plus either web or app components). Although more high-quality RCTs are needed, there is evidence supporting the effectiveness of mHealth approaches in those aged 50 years and older.
\end{abstract}

Keywords: aging, behavior change, mHealth, smartphones, technologies

Technology use among older adults has been increasing in tandem with the general trend of increasing technology use among the general population. Compared to 2012, when 53\% of older adults reported using the Internet, $59 \%$ of those aged 65 years and older in the United States now use it (Pew Research Center, 2014). Similarly, cell phone ownership among older adults has increased from $69 \%$ in 2012 to $77 \%$ in 2014 in the United States (Pew Research Center, 2014), with the latest statistics estimating that $80 \%$ of older adults (aged 65 years and older) in the United States now own some type of a cell phone, $47 \%$ of which are smartphones (Pew Research Center, 2017). The trends are similar in Europe, where $82 \%$ use the Internet, including $57 \%$ of those in the 55-74 age group (Eurostat, 2016). Cell phone ownership in Europe among older adults has been reported at $86 \%$ (Eurostat, 2016). Although data on smartphone ownership specifically among older adults in Europe are difficult to obtain, given comparable rates of smartphone ownership in the general population (67\% in Europe vs. $72 \%$ in the United States; Eurostat, 2016; Pew Research Center, 2016), it is reasonable to assume that a sizeable proportion of European older adults also use smartphones or other Internet-enabled mobile devices, a trend that is only expected to increase.

Like the older adults population, researchers have embraced technology as a welcome new platform for the promotion of health and health sustaining behaviors. A healthy lifestyle plays a vital role in reducing morbidity, functional limitations, and disability rates in older adults (Koster et al., 2007; Peel, McClure, \& Bartlett, 2005), also enhancing overall quality of life and increasing life expectancy (Arem et al., 2015; Drewnowski \& Evans, 2001; Moore et al., 2012). Physical activity (PA), sedentary behavior (SB), and sleep, in particular, are three lifestyle factors with the potential to

Elavsky is with the Faculty of Social Studies, Institute for Research on Children, Youth, and Family, Masaryk University, Brno, Czech Republic. Knapova, Klocek, and Smahel are with Masaryk University, Brno, Czech Republic. Address author correspondence to Steriani Elavsky at elavsky@fss.muni.cz. impact health and well-being in older adults (Buman et al., 2014; Mekary, Willett, Hu, \& Ding, 2009). These three behaviors can also be characterized by features that can be easily monitored with technology and potentially intervened upon through mobile platforms, such as smartphones or tablets. For example, in a recent study, Buman et al. (2016) reported on the development and process evaluation of an intervention utilizing a mobile application targeting sleep, PA, and SB among U.S. veterans with increased metabolic risk. The study showed that a mobile application targeting three behaviors (sleep, SB, and exercise) can be used in U.S. veterans with a metabolic syndrome and was found to be acceptable. The use of similar interventions supported by mobile apps is increasing, but whether such approaches for modifying and sustaining sleep, PA, and SBs will live up to the promise of expectations remains to be determined.

The term mobile health or mHealth (a subcomponent of eHealth) specifically denotes the use of mobile devices in support of medical and public health practice (World Health Organization, 2016a). In terms of health promotion and disease management, mobile devices have been incorporated into interventions involving both physical (in)activity and sleep, albeit not focused on behavioral outcomes per se. There have been several review articles of these types of studies specifically related to older adults. Chiarini, Ray, Akter, Masella, and Ganz (2013) conducted a comprehensive review of mobile health solutions that have been implemented for elderly people and patients with chronic conditions, resulting in a taxonomy of mHealth solutions (self health care management, assisted health care, supervised health care, and continuous monitoring) but not with a focus on behavioral outcomes. Other reviews have focused primarily on the use of web-based or remotefeedback interventions (without specific emphasis on mobile components; Beishuizen et al., 2016; Geraedts, Zijlstra, Bulstra, Stevens, \& Zijlstra, 2013), the use of eHealth or mHealth tools and other technologies in health promotion, primary care, or integrative medicine (Kampmeijer, Pavlova, Tambor, Golinowska, \& Groot, 2016; Knowles et al., 2016), or the use of body-worn sensors for 
monitoring PA (Taraldsen, Chastin, Riphagen, Vereijken, \& Helbostad, 2012) and smart devices for managing chronic disease in older adults (Kim \& Lee, 2017). Helbostad et al. (2017) provided an overview of mobile health applications and technologies for healthy and active aging, and Kruse, Mileski, and Moreno (2017) conducted a narrative review of mobile solutions focused on identifying barriers to and facilitators of adopting mHealth tools among older adults. All in all, these reviews have provided evidence of support for the effectiveness of using mHealth tools for disease management and health promotion in both healthy and chronically ill older adults.

More specifically, in terms of behavioral outcomes, two recent reviews focused on studies of older adults. Changizi and Kaveh (2017) published a systematic review of experimental studies (clinical trials and randomized control trials [RCTs]) published between 2012 and 2016 to assess the effectiveness of mHealth in changing health behaviors and improving disease recovery in older adults. The review had a broad focus on health behaviors ranging from PA, dietary habits, and sleep to medication adherence and mental health outcomes. Across 12 studies, the authors found support for the effectiveness of varied mHealth tools (cell phones, text messaging, and mobile apps) to improve varied health and behavior outcomes. The review excluded nonexperimental studies and did not include a specific focus on SB. In another recent paper, Muellman et al. (2018) published a review of studies using eHealth tools for PA among older adults. This review evaluated the effectiveness of eHealth interventions (i.e., those accessible via computer, handheld devices, telephones, smartphones, or tablets) and included experimental (RCT) or quasi-experimental studies comparing eHealth and non-eHealth PA interventions in adults aged 55 years and older. In 20 studies (across 25 publications), it was found that eHealth interventions can effectively promote PA in adults aged 55 years and older. The results were reported separately for web-based, telephone-based, and text-messaging interventions; however, the relative effectiveness of each type of intervention cannot be easily compared, given the uneven distribution of objective versus subjective measures of PA. The review did not include a specific focus on mobile interventions (e.g., through mobile apps) for health behaviors related to PA, such as SB and sleep.

In this study, we therefore set out to broadly review existing applications of mobile technologies targeting PA, SB, and sleep among adults aged 50 years and older. We lowered our age criterion below a level typically denoting older adults in order to accommodate the differences in the age ranges used across studies and to ensure that potentially promising mobile approaches applicable to older adults do not go unnoticed. Keeping up with intervention efforts in this area is essential in order to understand the efficacy and acceptability of interventions utilizing mobile platforms, given the sustained increases in mobile device ownership in all segments of the population, including older adults (Eurostat, 2016; Pew Research Center, 2016). Herein, we report on a systematic review of the literature targeting mHealth applications for PA, SB, and sleep among adults aged 50 years and older. Both experimental (RCT) and quasi-experimental studies and studies with single-group, pre/post designs are included in an effort to provide a broad overview of the different ways in which mobile technologies have been applied among those aged 50 years and older. In the review, we examine the prevalence and range of different mobile components used as part of PA, SB, and sleep interventions targeting adults aged 50 years and older and appraise the effectiveness of these interventions.

\section{Methods}

\section{Search Strategy}

In this study, we performed a systematic literature search of mHealth intervention studies in older adults (aged 50 years and older) targeting PA, SB, or sleep. Following the guidelines provided by the Preferred Reporting Items for Systematic Reviews and Meta-Analyses statement (PRISMA; Moher, Liberati, Tetzlaff, Altman, \& PRISMA Group, 2009), we conducted a search through electronic databases (Web of Science, SCOPUS, PubMed, and EBSCO_PsycARTICLES, PsycINFO, Medline, SPORTDiscus) to identify relevant studies, using the following search terms: ("mobile devices" OR "mobile health" OR mHealth OR m-health OR e-health OR eHealth OR telemedicine) AND ("physical activity" OR exercise OR sport OR "health behavior" OR "sedentary behavior" OR inactivity OR sitting OR sleep OR sleeping) AND ("older adults" OR elderly OR seniors). The same search was repeated twice - once with the term "smartphone" and second time with the terms "text messaging" OR "SMS" in place of ("mobile devices" OR "mobile health" OR mHealth OR m-health OR e-health OR eHealth OR telemedicine). No limitations were placed on date of publication or peer-review status, but an effort was made (when possible) to use filters for population age range within the database search engines. In such cases (PubMed, EBSCOPsycARTICLES, PsycINFO, Medline, SPORTDiscus), a search was performed without age restrictions and was repeated again with an age filter to identify studies targeting populations aged 50 years and older. The search was conducted from January-March 2017 and was subsequently updated in January 2018.

\section{Eligibility Criteria and Screening}

Following the automated searches, we removed duplicates and applied a two-stage process of article screening and selection. In the first stage, we reviewed the study titles, abstracts, and keywords to identify relevant articles based on the following eligibility criteria (these were used for acceptance into Stage 2, which involved screening of full-text articles using the same inclusion criteria):

a. The study involved a completed mobile intervention (defined as a treatment program delivered fully or partially by a mobile phone, smartphone, or tablet, either through applications, text messaging, or both). The use of wearable devices or sensors was acceptable as long as it was combined with a mobile platform to deliver elements of the intervention. Studies involving other eHealth approaches such as telemonitoring, telemedicine, telephone calls, exergaming, or use of websites only were excluded from this review, because these represent broader eHealth approaches (and have been recently reviewed by Muellmann et al., 2018). Studies involving (self)monitoring (e.g., via wearables) without any feedback provision or goal setting components were also excluded from this review, because these were not considered sufficient to constitute an active intervention (World Health Organization, 2016b).

b. The study targeted a population of adults aged 50 years and older. In cases in which the age range extended below 50, only studies with a mean age of participants of 50 years or older were included. Interventions targeting both healthy and clinical populations were considered. Psychiatric populations were the only population excluded from this review, because 
of the specificity of their needs in terms of managing behavioral outcomes.

c. The study reported on at least one of the behavioral outcomes of interest (PA, SB, and sleep) as either a primary or a secondary outcome, assessed either by self-report or objectively through sensors or other technology. Studies reporting on usability without behavioral data were excluded, as were case studies or reviews.

d. The study design was either experimental (RCT), quasiexperimental, or pre/post with at least one group of participants receiving the intervention.

e. The study was published in the English language.

The results of the search and screening are presented in the PRISMA flow diagram in Figure 1. After identification of relevant full-text articles, three reviewers (S. Elavsky, L. Knapova, and A. Klocek) read all of the studies, but each extracted data independently for one third of the articles. All extracted information was double-checked by the team. The following information was extracted: study design, country, study length/intervention duration, mean age, age range, number and type of participants, intervention type, mobile component, theory driven concept, available data on primary or secondary outcomes (PA, SB, and sleep behavior), and type of measurement of the outcomes. When possible, we extracted all relevant baseline and follow-up data (e.g., means, SDs/errors, pre/post correlations, effect sizes) to determine the feasibility of conducting a meta-analysis. The studies included in this review were heterogeneous in design, measurement, and reporting, precluding an effective use of meta-analysis; thus, results are reported in the form of a narrative review. Nonetheless, when available, effect size data were reported in Table 1 by study; effect size data by type of behavior overall are reported in Table 2 .

To assist with presentation of the data and to facilitate synthesis of the findings, harvest plots were generated to depict the key elements of the studies. In narrative reviews, harvest plots can be helpful in highlighting the most important findings across studies and illustrating selected study characteristics in an otherwise diverse group of studies. Specifically, we generated four separate plots (one with results for RCTs and PA, one for RCTs and SB, one for pre/post studies and PA, and one for pre/post studies and SB) to illustrate significant findings across the studies (not effect sizes). Categories depicted in the harvest plot matrix include mobile intervention component (i.e., text messaging, mobile application, or hybrid intervention with a mix of components) in the columns, and population type (i.e., healthy, special) in the rows. For each study, we specified the type of findings (no effect of the intervention, positive, or negative outcome) related to indicators of overall PA (i.e., self-reported MET hours per week or accelerometer derived counts per minute) or estimates of moderate to vigorous PA (MVPA) if overall PA data were not available. Studies with objective behavioral outcome measures were indicated with full (dark) bars and studies with subjective behavioral outcome measures with empty (white) bars. The height of the bar indicates the study sample size. Studies that explicitly reported using theory to guide intervention content were indicated by the letter " $T$ " below the specific bar. Each bar was also annotated with a number above, which corresponds to the number of the study in our review (from 1 to 52) and in Table 1 . Table 1 contains results related to physical

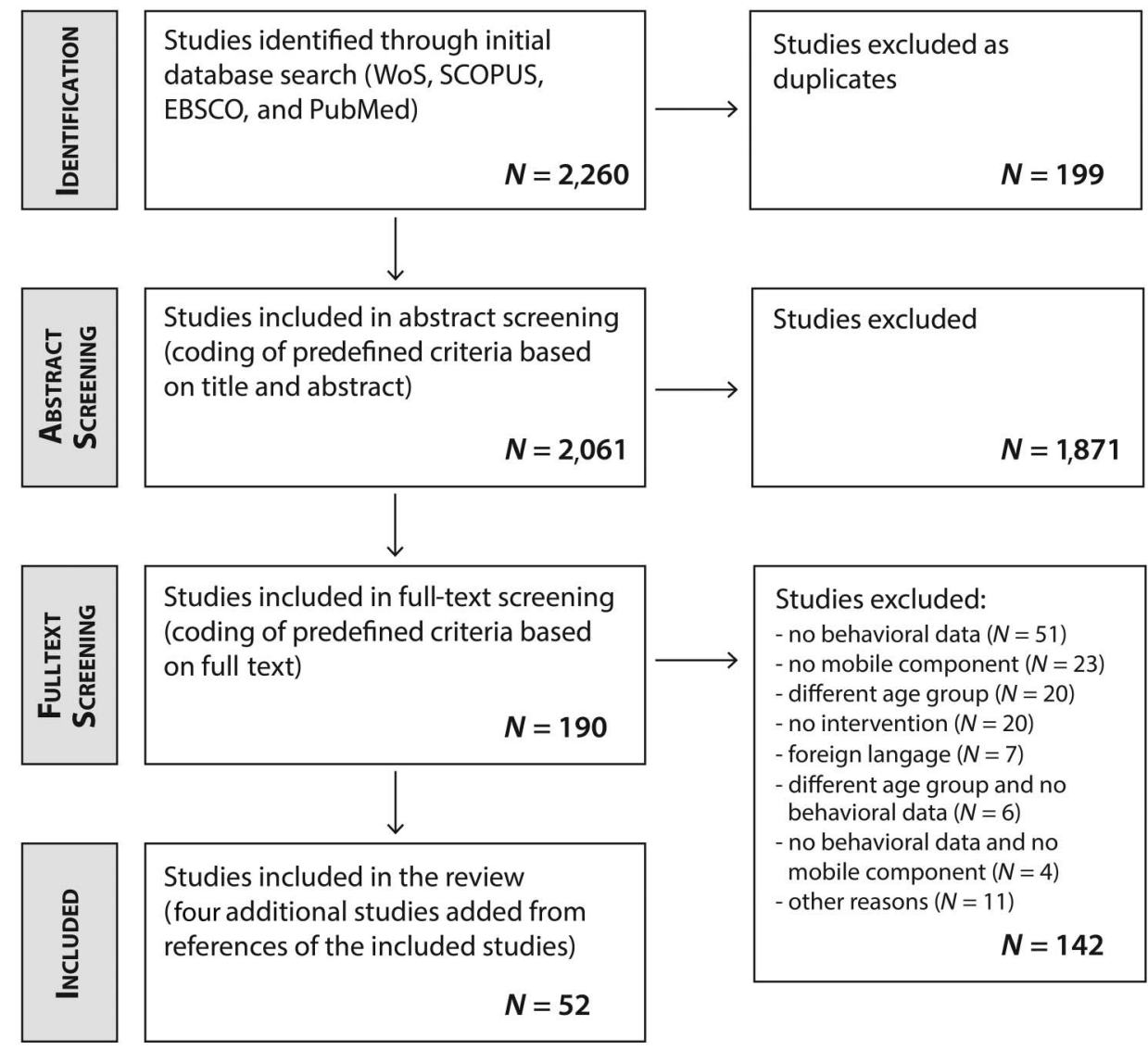

Figure 1 - Diagram depicting the flow of studies in different phases of the systematic review. 


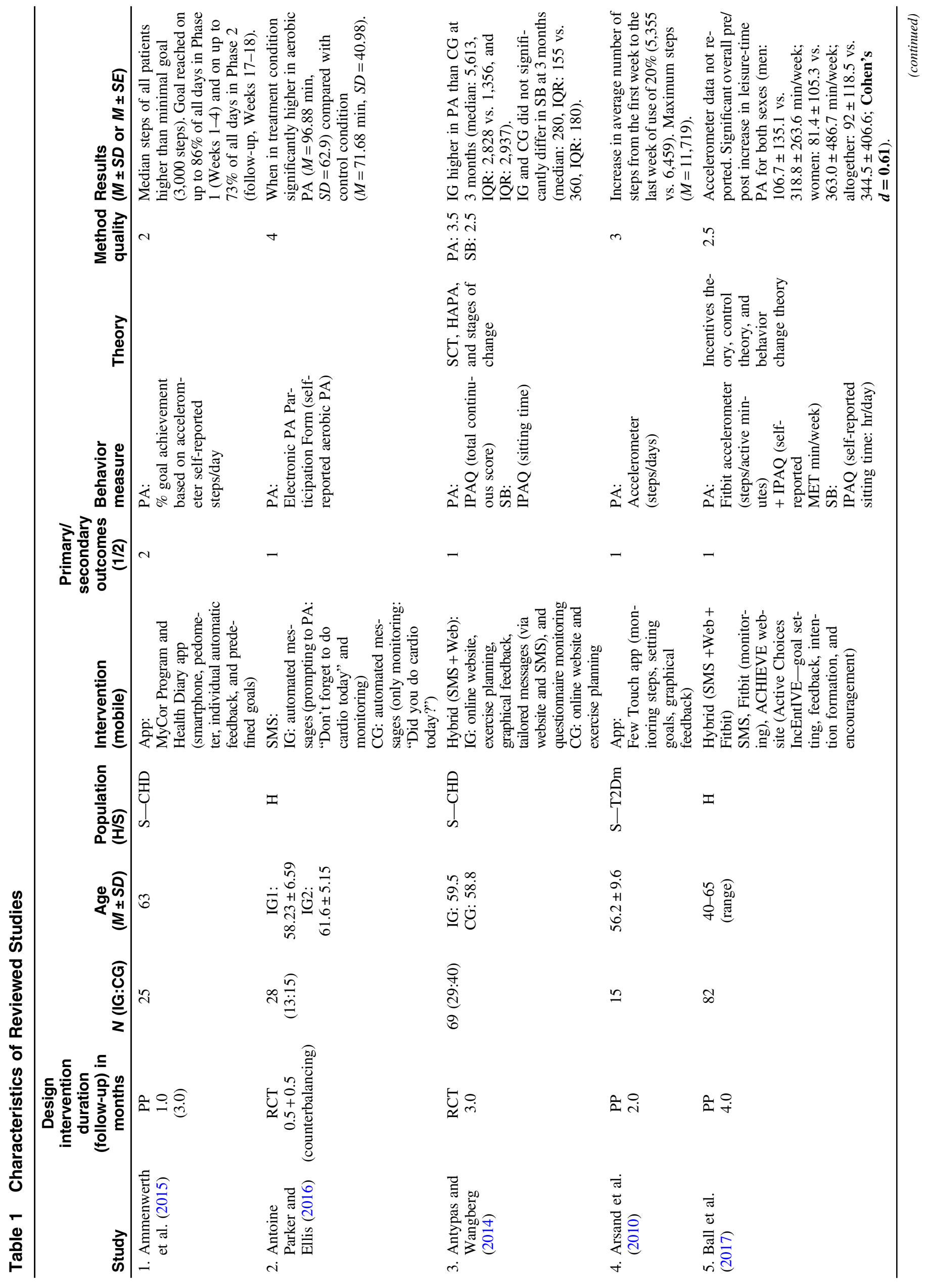




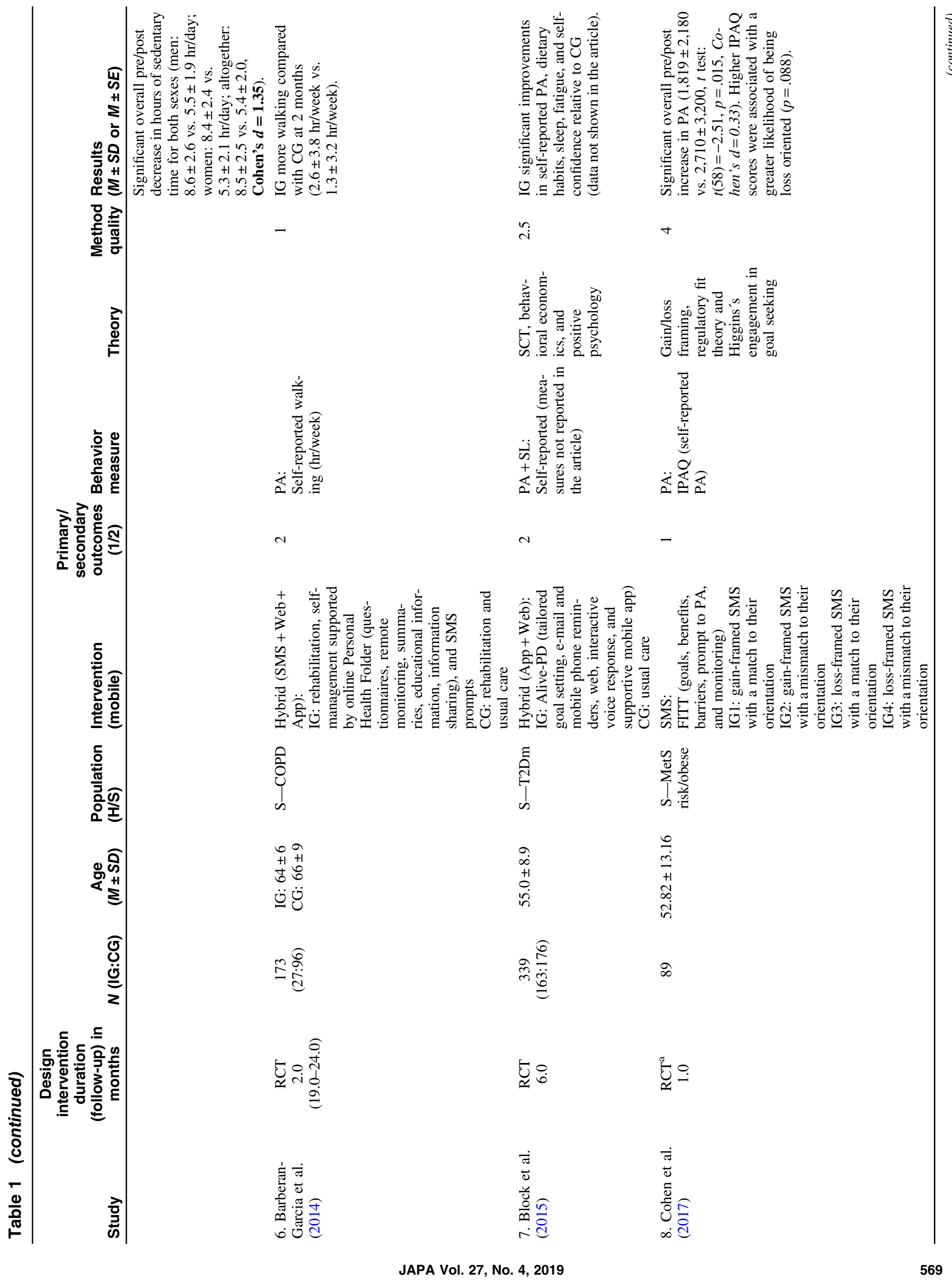




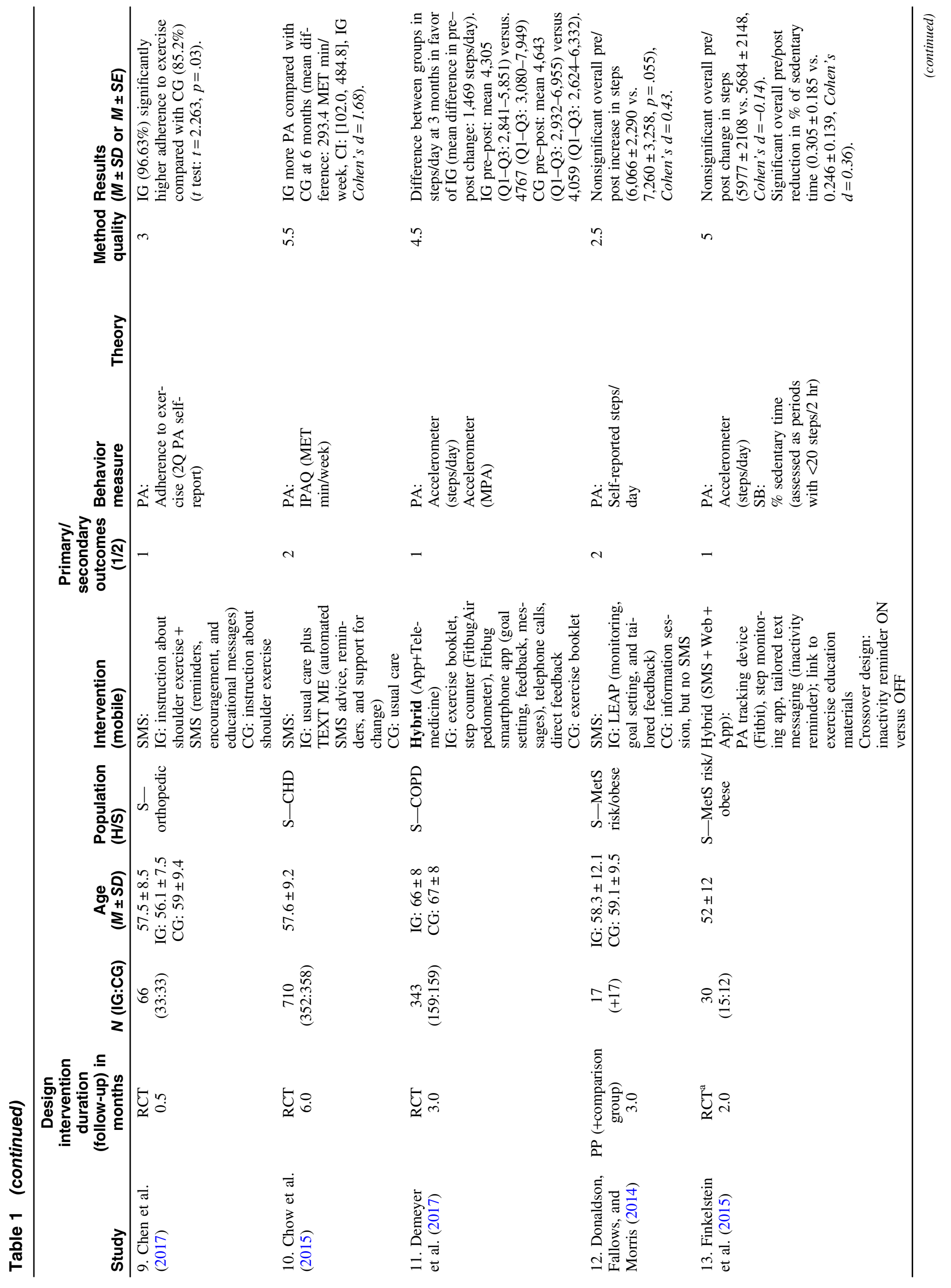




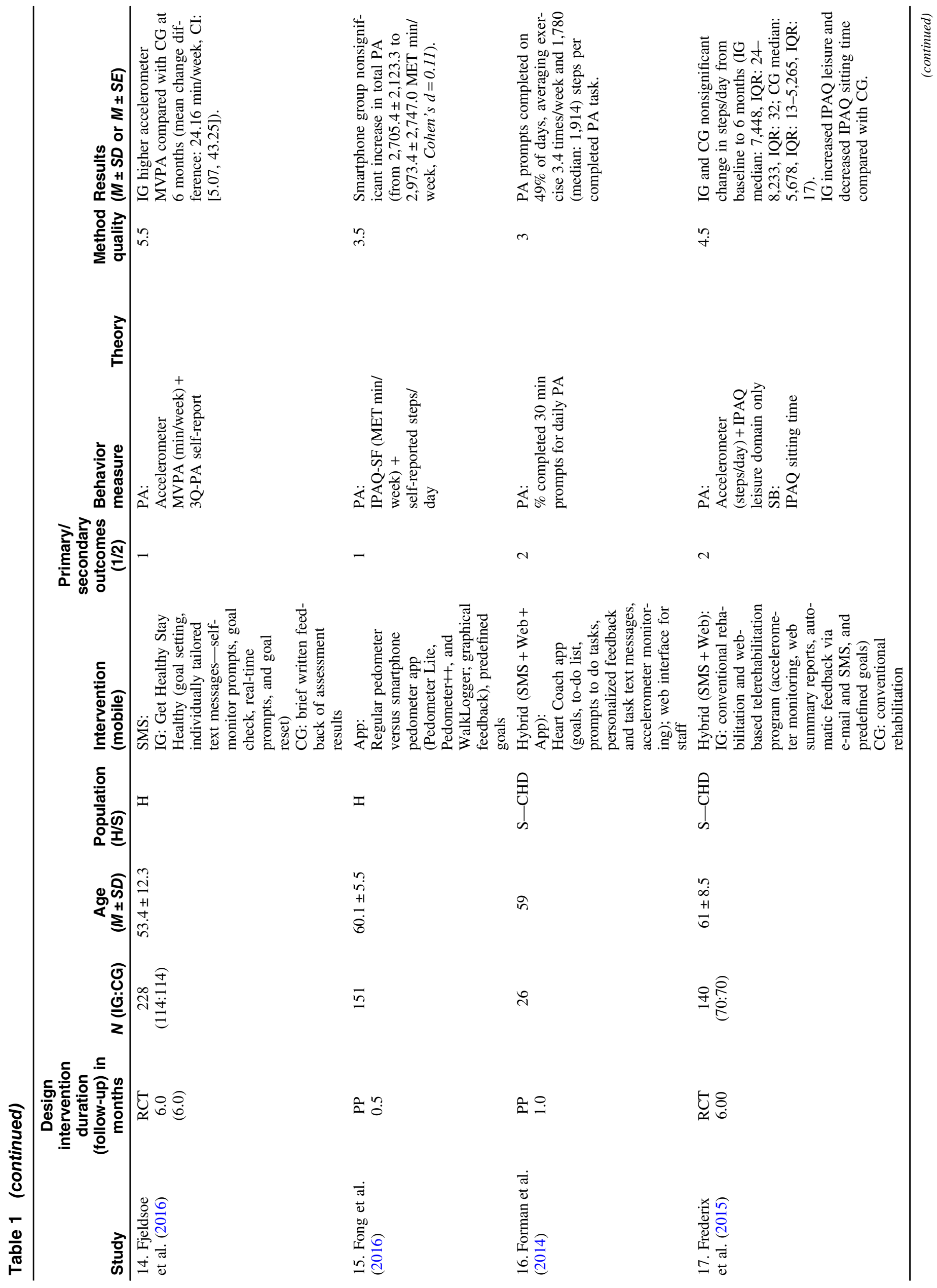




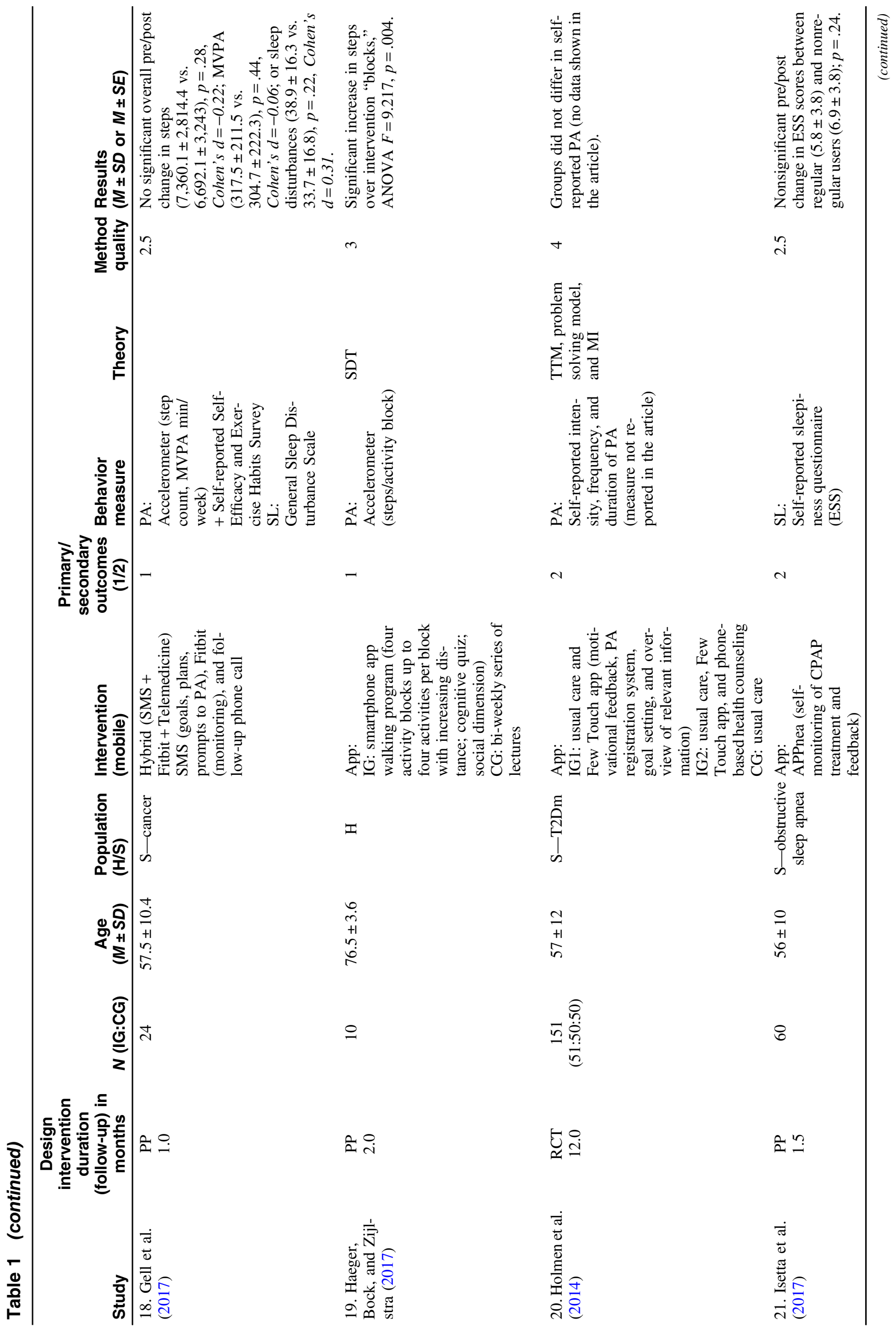




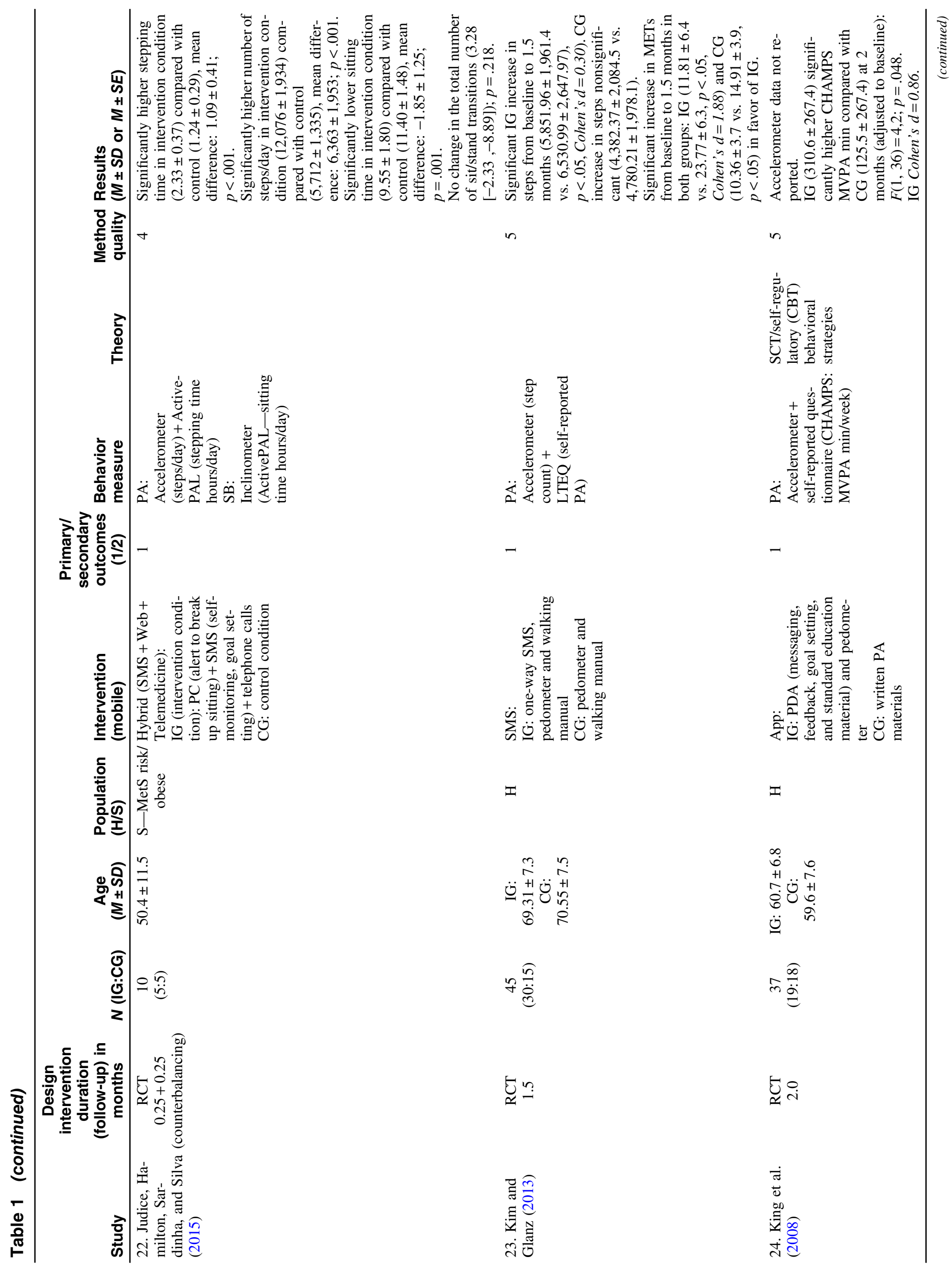




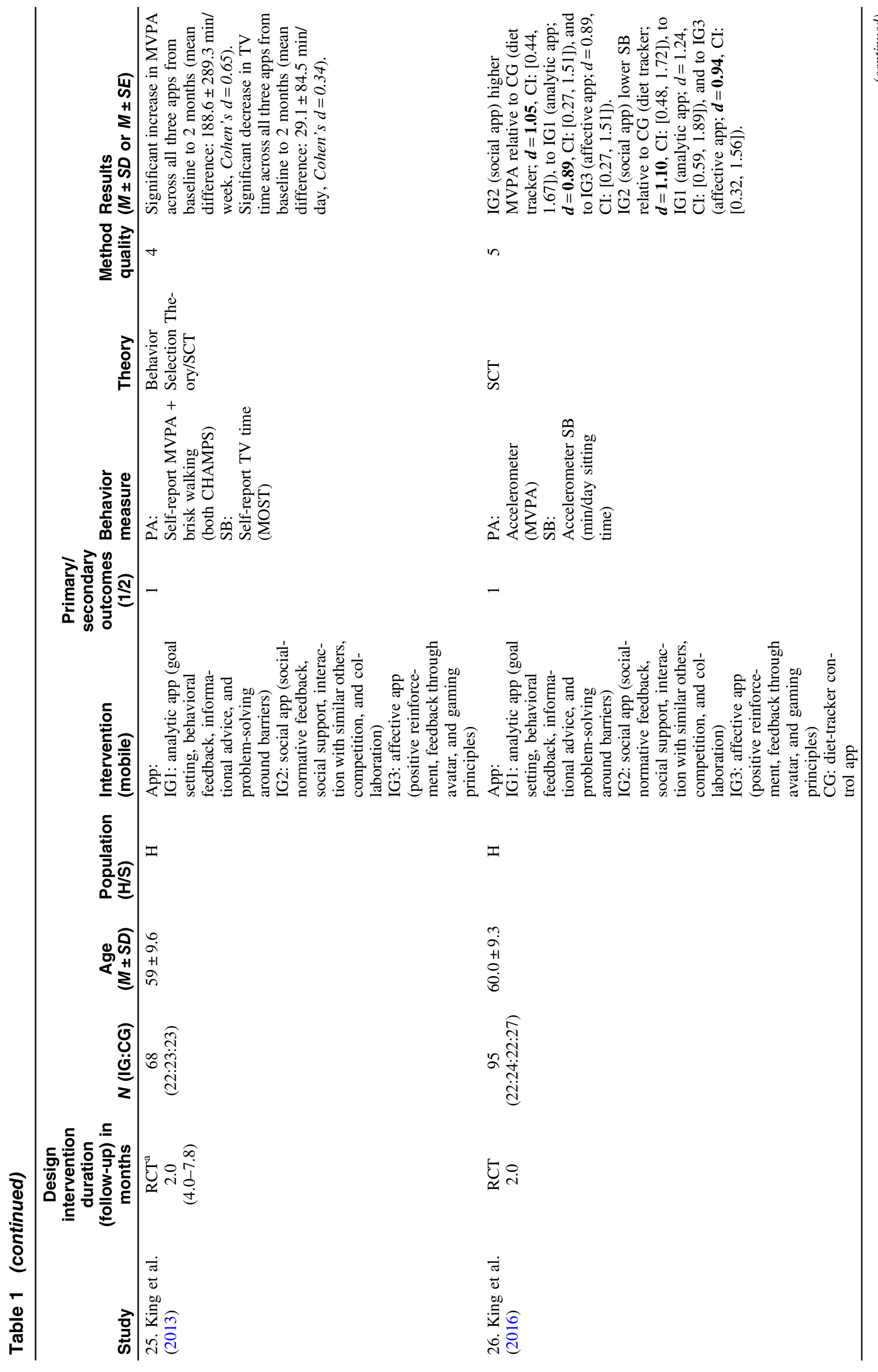




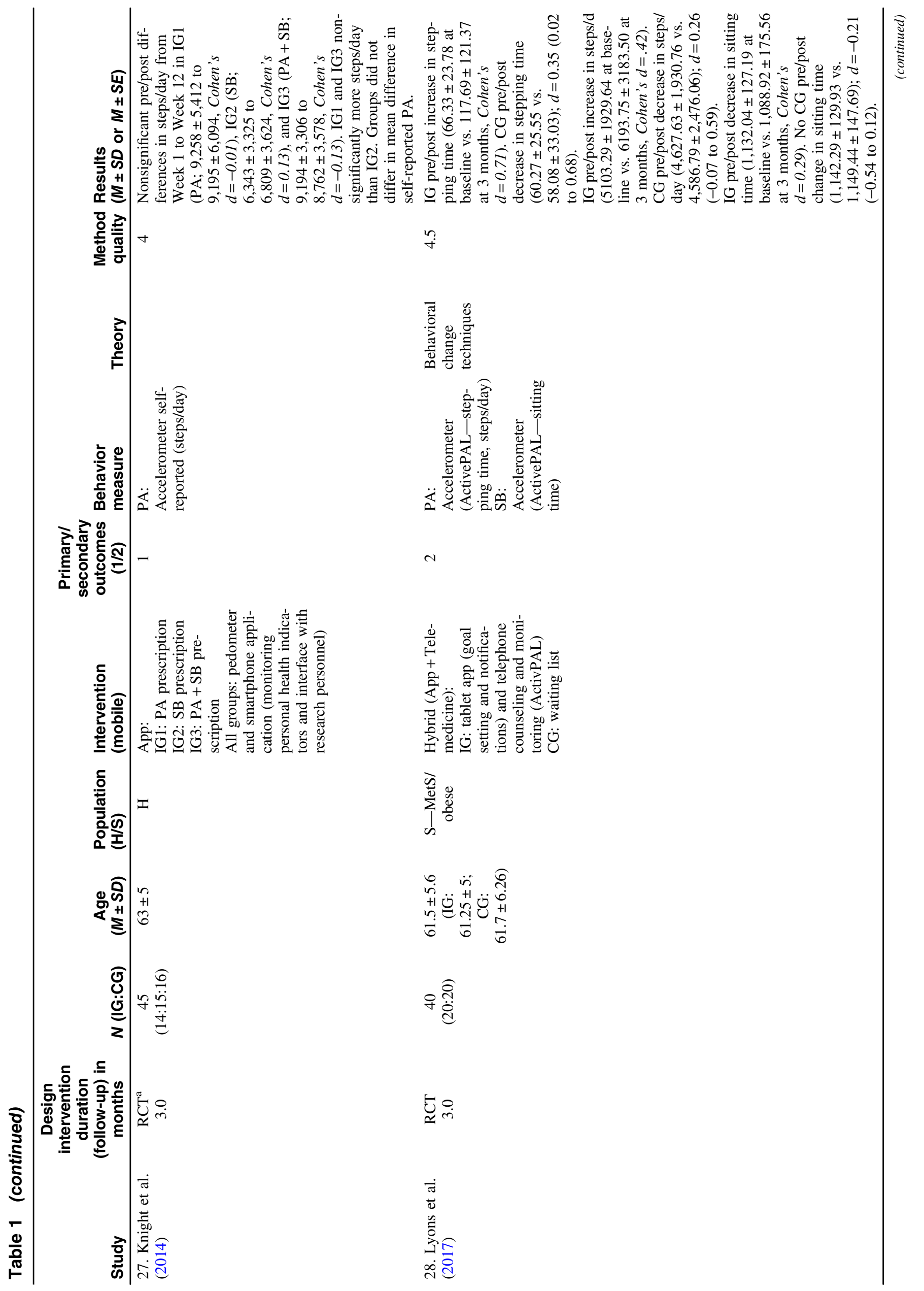




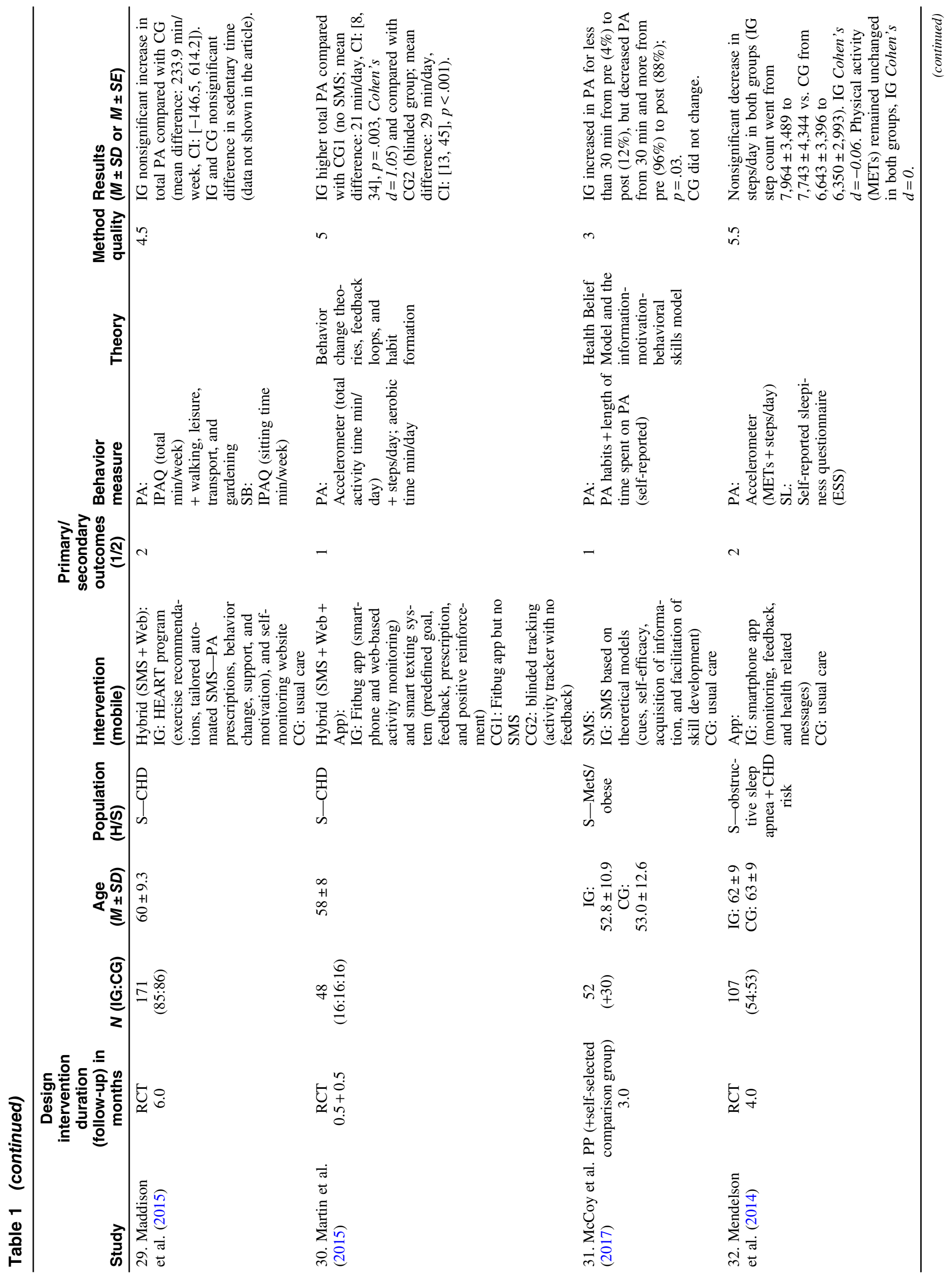




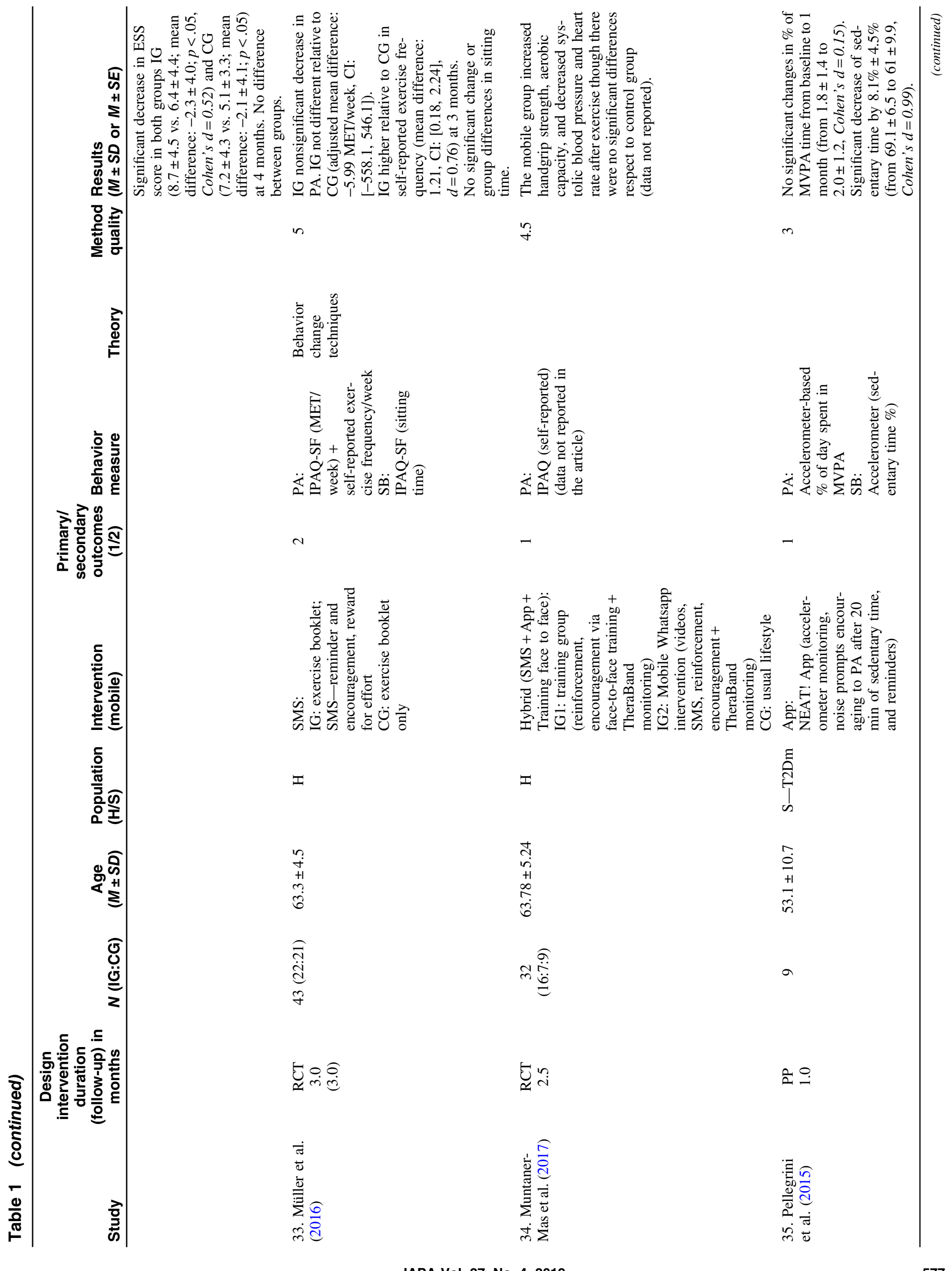




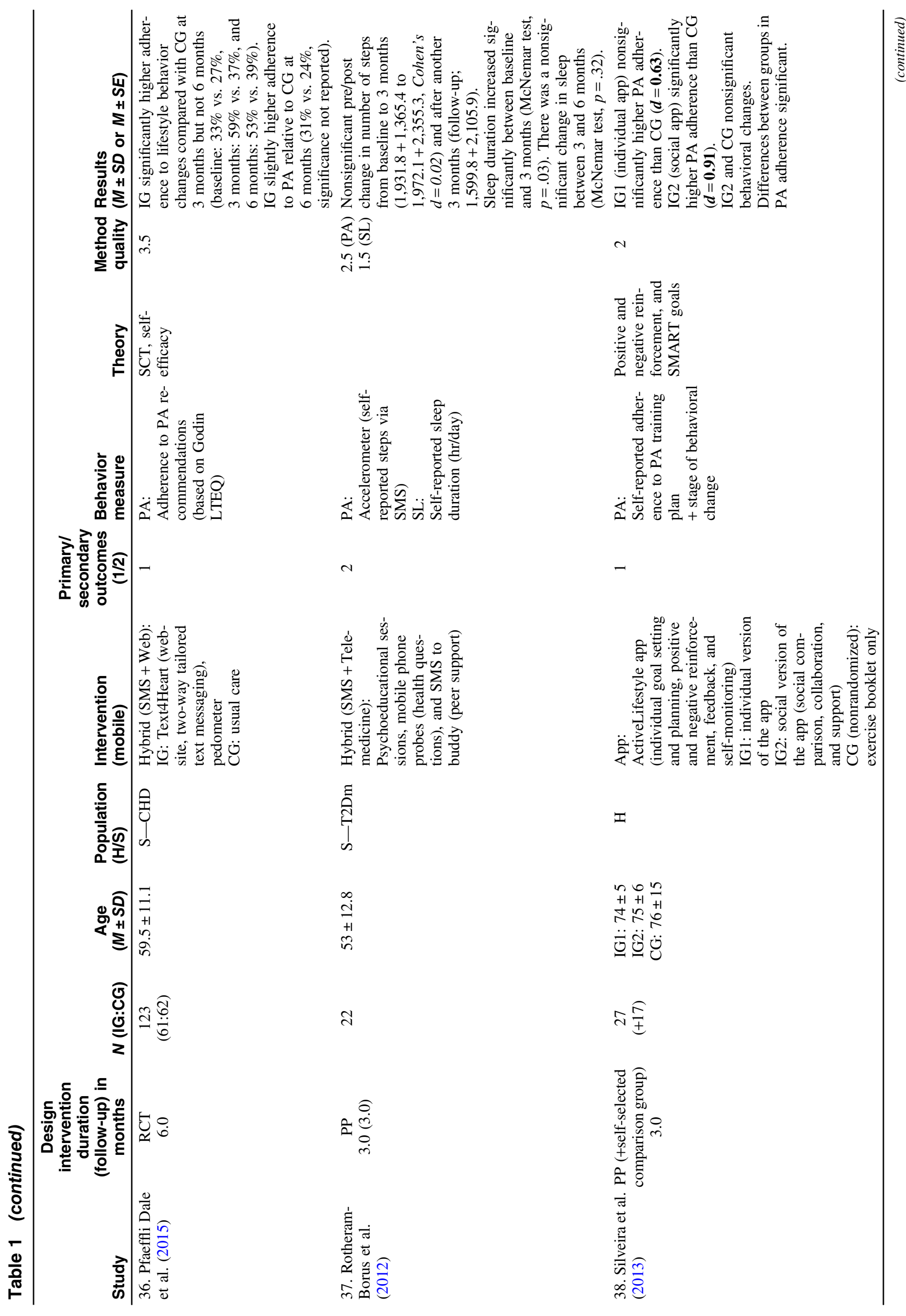




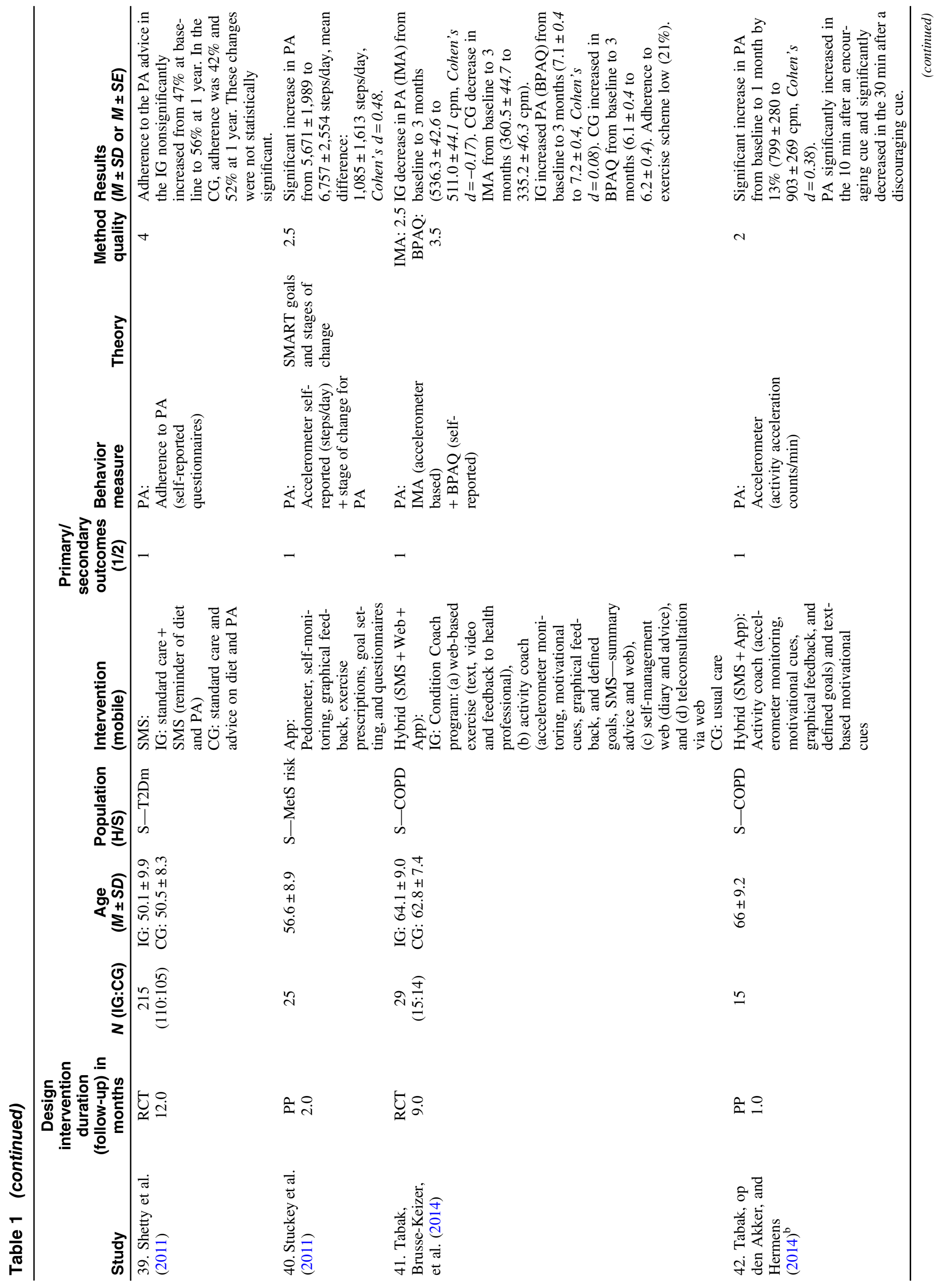




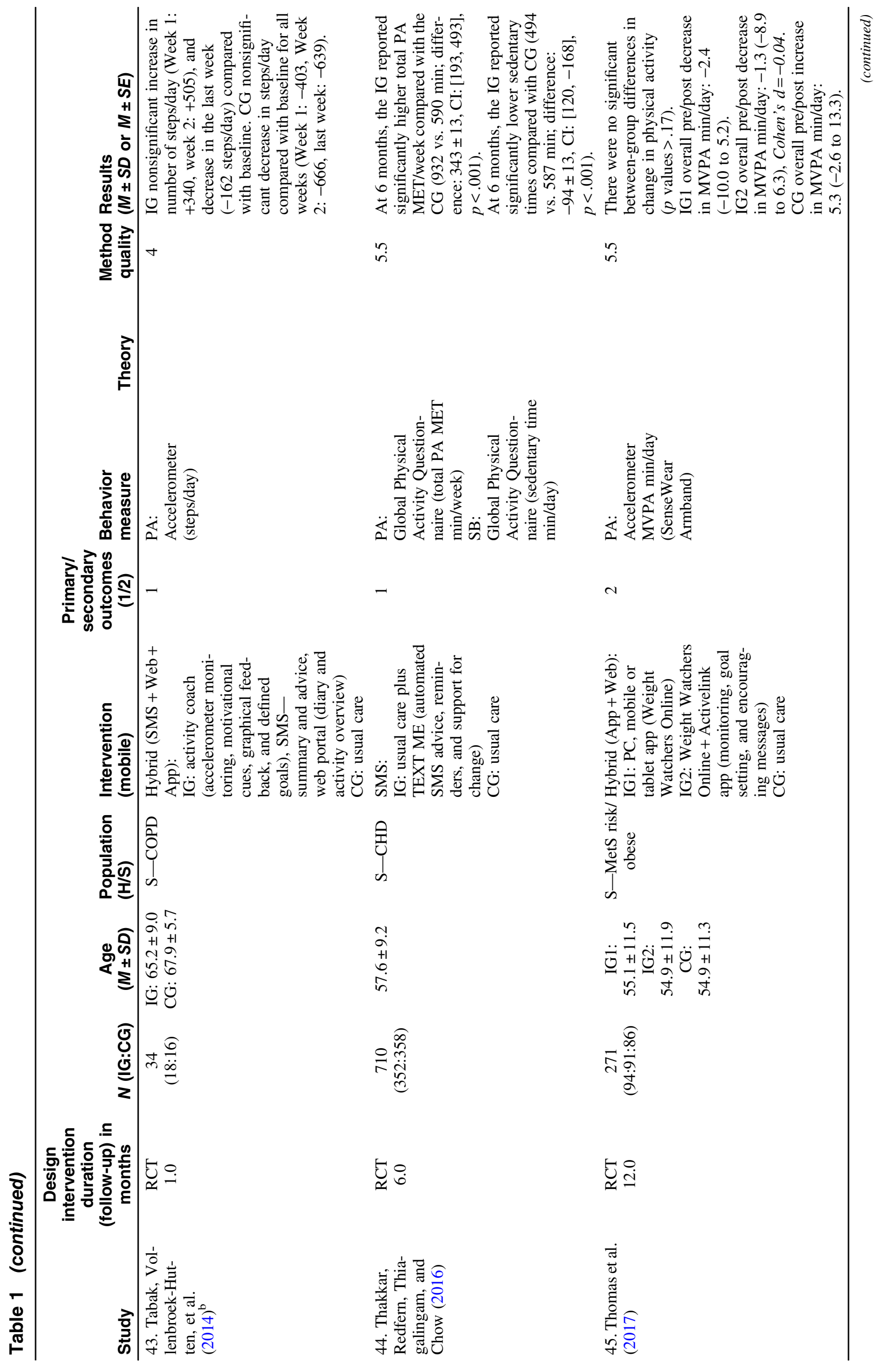




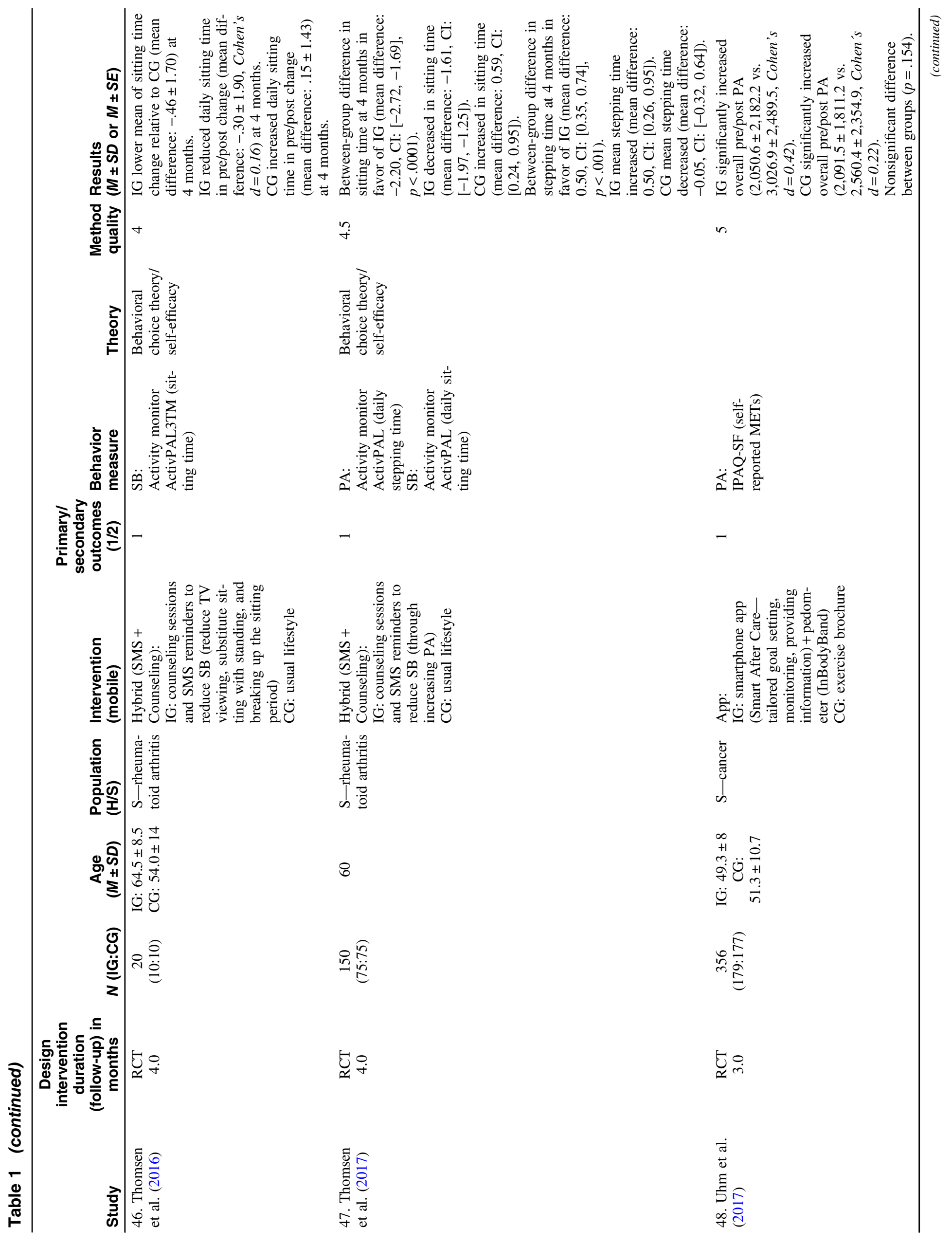




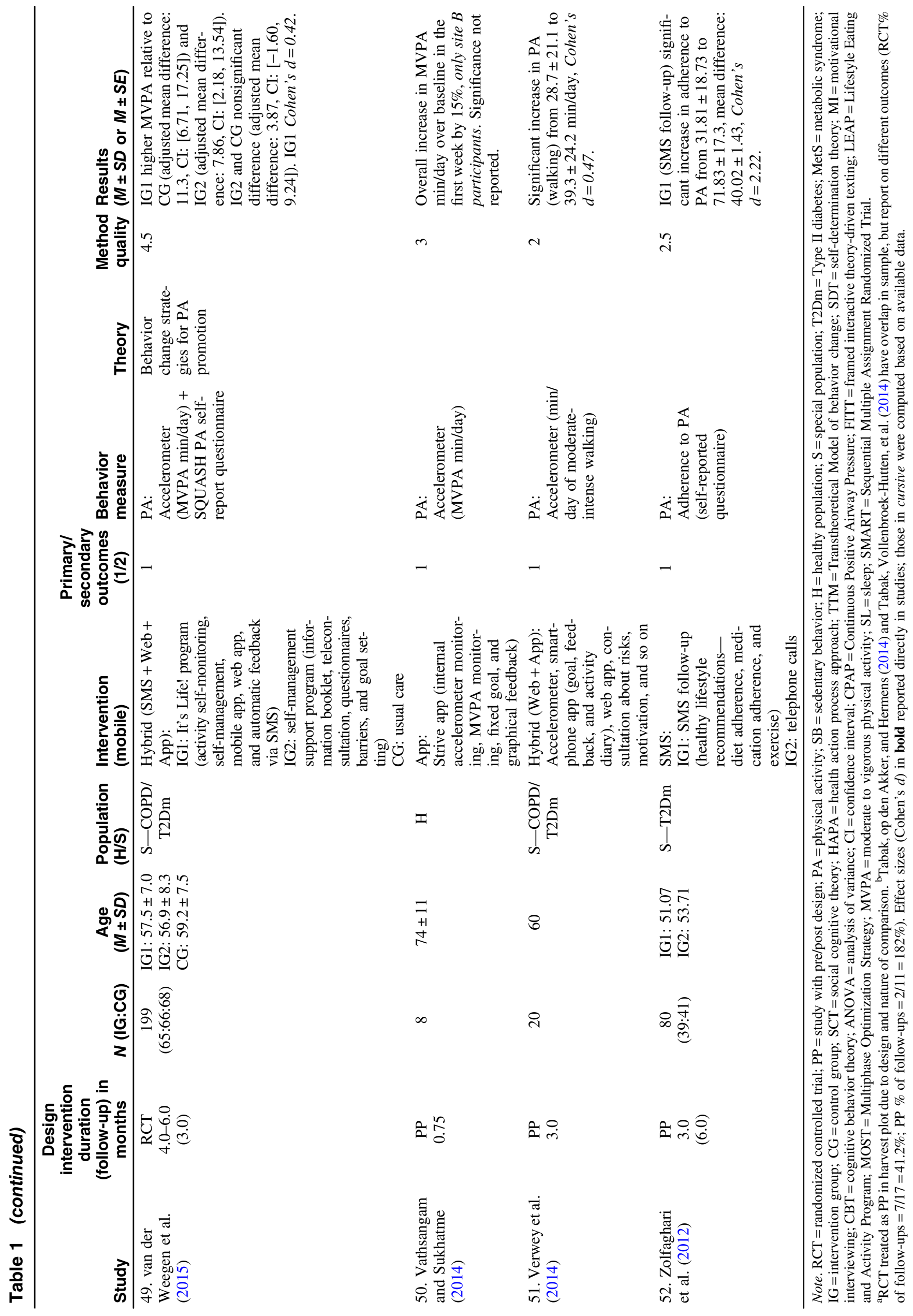


Table 2 Overview of Methodological Quality Ratings Across Reviewed Studies, Overall Effect Size Data, and Intervention Components Used

\begin{tabular}{|c|c|c|c|c|c|c|}
\hline \multirow[b]{2}{*}{ Study category } & \multicolumn{3}{|c|}{ Methodological quality } & \multicolumn{3}{|c|}{ Effect size } \\
\hline & $N$ & $M \pm S D$ & Range & $n$ & Unweighted Cohen's d & Weighted Cohen's d \\
\hline PA & 50 & $3.74 \pm 1.16$ & $1-5.5$ & 26 & 0.49 & 0.69 \\
\hline SB & 14 & $4.18 \pm 0.93$ & $2.5-5.5$ & 7 & 0.66 & 0.77 \\
\hline \multirow[t]{2}{*}{ SL } & 5 & $3.00 \pm 1.73$ & $1-5.5$ & 2 & 0.42 & 0.46 \\
\hline & & & & \multicolumn{3}{|c|}{${ }^{\mathrm{a}}$ Behaviors targeted by type of intervention } \\
\hline App intervention & 16 & $3.56 \pm 1.12$ & $1-5.5$ & & $\mathrm{PA}=15 ; \mathrm{SB}=3$ & \\
\hline SMS intervention & 12 & $4.13 \pm 1.17$ & $2.5-5.5$ & & $\mathrm{PA}=12 ; \mathrm{SB}=2$ & \\
\hline Hybrid intervention & 24 & $3.58 \pm 1.19$ & $1-5.5$ & & $\mathrm{PA}=23 ; \mathrm{SB}=9$ & \\
\hline Overall & 52 & $3.70 \pm 1.17$ & $1-5.5$ & & & \\
\hline
\end{tabular}

Note. Studies targeting PA only $=33 ; \mathrm{PA}+\mathrm{SB}=13 ; \mathrm{PA}+\mathrm{SL}=4 ; \mathrm{SB}$ only $=1 ;$ SL only $=1$. Cohen's $d$ for available studies $(n)$-unweighted and weighted by sample size. $\mathrm{PA}=$ physical activity; $\mathrm{SB}=$ sedentary behavior; $\mathrm{SL}=$ sleep.

${ }^{\text {a } T o t a l ~ e x c e e d s ~} 52$ due to some interventions targeting multiple behaviors.

Pre/post studies on physical activity

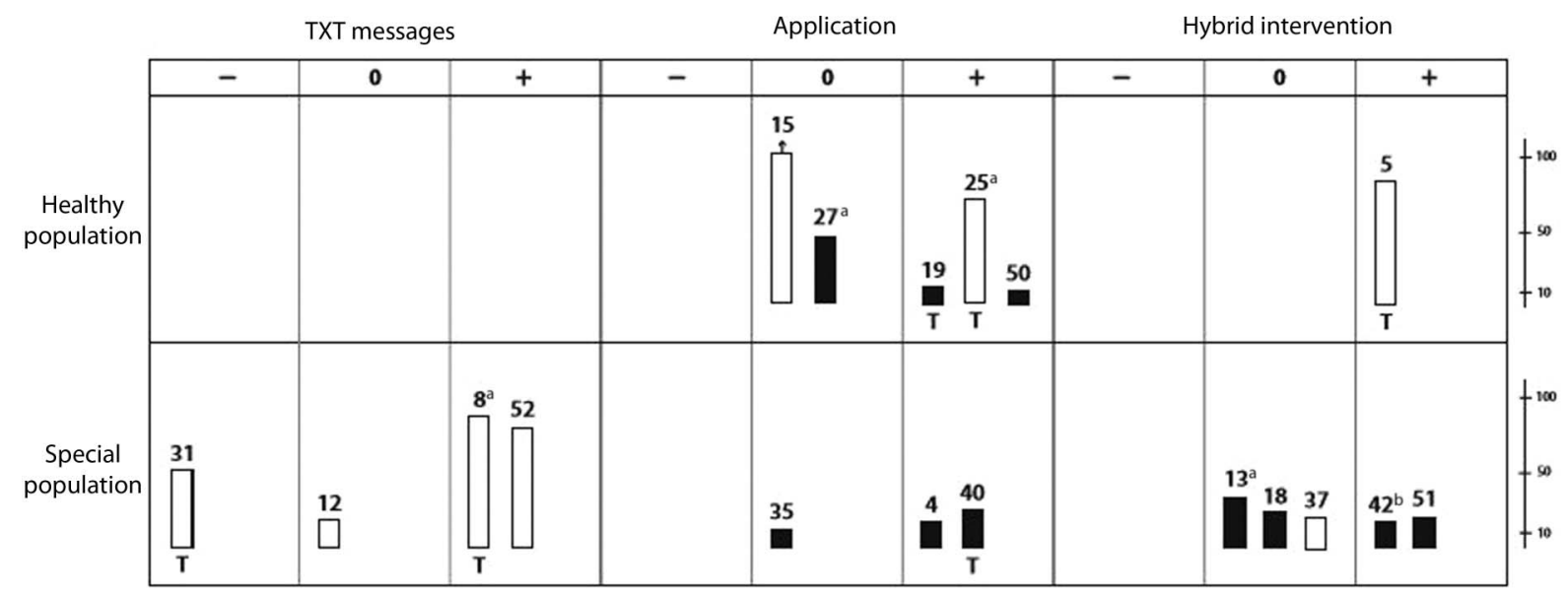

RCT studies on physical activity

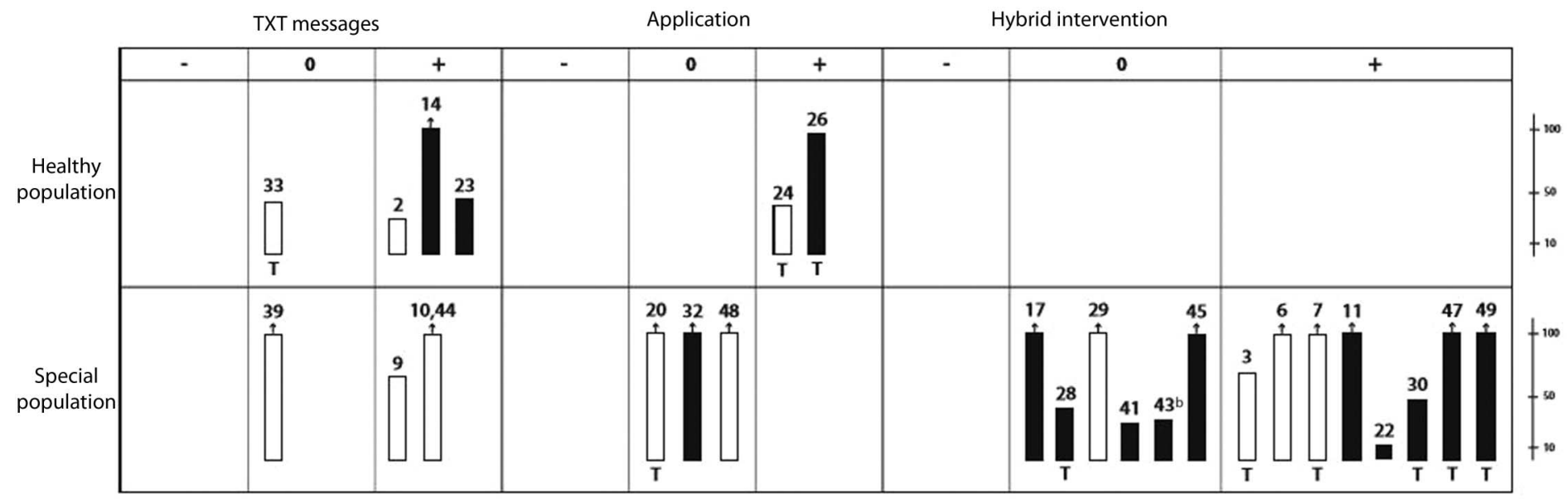

Figure 2 - Harvest plots depicting results of studies targeting physical activity. Each study is represented by a bar labeled with the study number (according to Table 1). The bar is placed in the corresponding cell according to the type of intervention (columns), study population (rows), and effect (negative, null, or positive; columns). The size of each bar corresponds to sample size; bars with an arrow pointing upward have $N>100$. Studies with objective outcome measures are indicated with full (black) bars, and studies with subjective outcome measures are indicated with empty (white) bars. The letter "T" below the bar indicates that the intervention was theory based. ${ }^{\text {a }} \mathrm{RCT}$ treated as pre/post in harvest plot due to design and nature of comparison. ${ }^{\mathrm{b}}$ Tabak, op den Akker, and Hermens (2014; \#42) and Tabak, Vollenbroek-Hutten, et al. (2014; \#43) have overlaps in sample, but report on different outcomes. Chow et al. (2015; \#10) and Thakkar, Redfern, Thiagalingam, and Chow (2016; \#44) report data from the same study and are depicted as a single bar in the harvest plot. TXT $=$ text; RCT $=$ randomized control trial. 
(in)activity outcomes depicted in the harvest plots as well as additional outcome measures when applicable (e.g., self-reported light PA, transport PA, or walking). For cases in which data were available, effect sizes for mobile intervention groups were reported in Table 1. Effect sizes in bold represent data reported directly in studies. Effect sizes in italics were computed based on available data $(d=0.2,0.5$, and 0.8 correspond to small, medium, and large effects, respectively).

The methodological quality of the studies was evaluated using six predetermined criteria adopted and modified from previous research (Ogilvie et al., 2008): representativeness ( $1=$ representative sample plus minimum $60 \%$ response rate; $0.5=$ nonrepresentative sample with $n>100 ; 0=$ nonrepresentative sample with $n<100$ ), randomization ( $1=$ randomized design; $0.5=$ nonrandomized design but at least two comparison groups; $0=$ pre/post single group design), comparability $(1=$ groups comparable or analysis adjusted for baseline differences; $0.5=$ baseline values reported but not adjusted for in analysis; $0=$ pre/post single-group design or baseline values/adjustment not reported), credibility of data collection $(1=$ objective or validated self-report measures; $0=$ nonvalidated or unknown measures $)$, attrition rate $(1=$ maximum $30 \%$; $0.5=$ maximum $50 \% ; 0=$ attrition $>50 \%$ ), and attributability to intervention $(1=$ design allows the evaluation of a specific mobile component-i.e., single-component intervention or groups differ only in the presence of a mobile component; $0.5=$ mobile component as main component but unclear description of other aspects-i.e., unable to completely rule out influence of other components; $0=$ mobile component utilized but unable to separate its effects from other components-i.e., multicomponent interventions). Thus, the methodological quality score could range from 0 to 6 . Studies were evaluated independently and assigned scores by two reviewers, and the team (S. Elavsky, L. Knapova, and A. Klocek) resolved differences in scoring based on group consensus (only $<5 \%$ discrepancy in ratings).

\section{Results}

The searches retrieved a total of 2,260 published articles. After removing duplicates, 2,061 articles remained, of which 1,871 were removed based on abstract screening. In the full-text screening phase, 190 articles were screened and four new articles were identified through references. Of these, 142 were excluded; the reasons for exclusion were mainly containing no behavioral data (51), no mobile component (23), different age group (20), and no intervention or incomplete intervention (20) (see Figure 1). After the selection process, 52 articles remained (details of all studies can be found in Table 1).

\section{Study Design}

Out of the 52 studies, 34 were RCTs and 18 had quasi-experimental (i.e., nonrandomized comparison group, $n=3$ ) or pre/post singlegroup study design $(n=15)$. The mean intervention duration was 3.5 months (range $=0.5-12$ ).

\section{Samples}

The mean number of participants was $N=114$ (range $=8-710$ ). The mean age of the participants was 59.6 years (range $=50-76.5$ ). Only three studies explicitly targeted populations 60 years and older (Heager, Bock, \& Zijlstra, 2017; King et al., 2008; Silveira et al., 2013); the remaining studies used lower age cutoffs to designate older adults (e.g., 50 years and older or 55 years and older) or targeted broader age groups (e.g., 18-65 or 40-65). Fourteen studies targeted healthy populations and 38 involved special populations (heart disease or cardiovascular disease risk factors [9], diabetes [7], COPD [5], COPD or diabetes [2], metabolic syndrome/obese [7], obstructive sleep apnea [2], cancer [2], rheumatoid arthritis [2], orthopedic [1], and primary care patients [1]). Seventeen of the studies were conducted in the United States, five in the Netherlands, four in Australia, three in Canada, two each in the UK, New Zealand, Spain, Denmark, and Norway, and the remaining studies represented Germany, Austria, Portugal, Switzerland, Finland, Iran, South Africa, South Korea, India, Malaysia, Taiwan, and Hong Kong. One study included participants from several European Union countries.

\section{Behaviors}

The majority of studies targeted only PA (33), and 13 targeted both PA and SB (of these, one study targeted interruptions in sedentary activity through a smartphone app but reported outcomes related to both PA and SB; Pellegrini et al., 2015). One study targeted only SB. One study targeted only sleep outcomes and four studies targeted both PA and sleep. PA, SB, and sleep were the primary outcomes in 37 studies and were secondary outcomes in 15 studies. In 23 of the studies, the behaviors were assessed subjectively through self-report; 17 studies contained objective data (although four of them relied on participants to self-report accelerometerbased steps); and 12 studies reported some combination of both subjective and objective assessments. The most commonly reported PA outcomes were MET hours per week (based on IPAQ, nine studies), other self-reported levels of PA or walking (11 studies), steps per day (11 studies), and other actigraphy data (16 studies). Several studies reported alternative PA outcomes such as adherence rates to PA prompts or PA recommendations/plans (six studies), goal achievement (one study), exercise frequency (one study), and stage of change (two studies). SB was assessed by self-report as time spent sitting (based on IPAQ, five studies), selfreported sedentary time (two studies), or sedentary time as determined by Fitbit (two studies) or accelerometry (six studies). Sleep outcomes were assessed by self-report (five studies-with two assessing sleepiness, one assessing sleep disturbance, one assessing sleep duration, and one not reporting the type of sleep measure), although studies targeting patients with obstructive sleep apnea also assessed apnea parameters and CPAP (Continuous Positive Airway Pressure treatment) usage objectively through telemedicine.

\section{Theories}

Based on information provided in the articles, 19 studies were theory driven. Some studies used a combination of different theories. The most common theories guiding the interventions were Social Cognitive Theory (6), Transtheoretical Model of Behavior Change (1), Health Belief Model (1), Health Process Action Approach (1), and Self-Determination Theory (1); the remainder of the studies mentioned behavior change theory/behavior change techniques broadly (4), or used another theory (3). In two studies, Sequential Multiple Assignment Randomized Trial goal setting principles and reinforcement were listed as behavior change techniques; the remainder (33) made no explicit mention of a guiding theory. 


\section{Mobile Components}

In terms of mobile intervention components, 12 studies used text messaging only, 16 used mobile apps, and 24 used a combination of text messaging, mobile app, web, or other components. Among those with text messaging, some used text-messaging programs (e.g., TEXT ME, TEXT4HEART) and others used normal SMS. These interventions consisted of using SMS as reminders, for providing advice/information, for support, for self-monitoring through SMS, even for goal setting. Studies with mobile apps used applications that were either commercially available (such as the Fitbit app) or were designed specifically for the study (e.g., ActiveLifestyle app, NEAT!, or MyCor Health Diary app). The app-facilitated interventions typically consisted of a tailored PA or exercise plan and self-monitoring as key intervention components, sometimes accompanied by objective monitoring through a wearable sensor such as an accelerometer, pedometer, or Fitbit. Other app features included provision of feedback (automatic/tailored), setting of individual or group goals, providing emotional support or motivational cues, prompts encouraging activity after periods of inactivity, and, in some cases, social components (chats, comparison with others). Hybrid interventions included different combinations of a mobile component (SMS, App, or both) and web components such as online web portal or reminder e-mails. Some hybrid interventions had multiple other components, including personal interaction or personal/telephone counseling.

\section{Methodological Quality}

On a scale ranging from 0 to 6 , the average methodological quality of the studies was moderate $(M=3.70, S D=1.17)$, ranging from 1 to 5.5. The methodological quality of the studies tended to be lowest in studies targeting sleep and highest in studies involving SB and SMS interventions (see Table 2).

\section{Effectiveness of Interventions}

The effectiveness of the interventions was evaluated separately for studies with quasi-experimental (nonrandomized comparison group) or pre/post single-group design and for RCTs. In cases in which the reported data allowed it (46 out of 52 studies), the study results were depicted in the accompanying harvest plots (Figure 2 depicts 45 of 50 studies on PA; Figure 3 depicts all 14 studies on SB). Studies involving sleep outcomes are reported in the text only (and in Table 1). Some studies with RCT design did not allow us to evaluate the effectiveness of interventions with mobile components compared with nonmobile alternatives; thus, four studies with RCT design were treated as pre/post studies in the harvest plots: \#8 (Cohen et al., 2017), \#13 (Finkelstein, Bedra, Li, Wood, \& Ouyang, 2015), \#25 (King et al., 2013), and \#27 (Knight, Stuckey, \& Petrella, 2014).

Physical activity. Altogether, 50 out of the 52 studies included in this review targeted PA; 17 had quasi-experimental or pre/post single group-design, and 33 were RCTs. Depicted in the harvest plot (Figure 2) are a total of 45 studies. The top harvest plot depicts 14 out of the 17 quasi-experimental or pre/post studies plus four RCTs studies assessed only for pre/post change. The lower plot depicts 27 out of 33 RCTs. Studies not depicted in the harvest plot due to atypical or incomplete outcome data are reported in Table 1.

Out of 21 studies evaluated for pre/post change (i.e., 17 quasiexperimental or pre/post studies and four RCTs evaluated for pre/post change only), 13 provided some evidence of effectiveness to increase PA. Specifically, eight pre/post studies demonstrated increases in PA based on: mobile app-based steps per day in a 6-month intervention with a smartphone app (\#4; Arsand, Tatara, Ostengen, \& Hartvigsen, 2010), self-reported steps per day following an 8-week smartphone-enabled intervention (\#40; Stuckey et al., 2011), accelerometer-derived steps in a 2-month smartphone app walking program (\#19; Haeger, Bock, \& Zijlstra, 2017) and a 4-month hybrid intervention (\#5; Ball et al., 2017), counts per minute following a 4-week telerehabilitation intervention utilizing a smartphone app (\#42; Tabak, op den Akker, \& Hermens, 2014), accelerometer-derived moderate PA minutes following an 8- to 12-week intervention utilizing a smartphone app (\#51; Verwey et al., 2014), accelerometer-derived MVPA across a 3-week smartphonebased pilot intervention (\#50; Vathsangam \& Sukhatme, 2014), and self-reported adherence to exercise in a 3-month text-messaging intervention (\#52; Zolfaghari et al., 2012). Three additional studies (not depicted in the harvest plot) reported improvements in diverse PA outcomes such as goal achievement (\#1-a telemonitoring intervention utilizing a smartphone app; Ammenwerth et al., 2015), adherence to training plan and stage of behavior change following a tablet-based strength training program (\#38; Silveira et al., 2013), and adherence to PA prompts (\#16-a 30-day feasibility study utilizing a smartphone app in cardiac rehabilitation; Forman et al., 2014). Additionally, in two randomized studies (\#8 and \#25) self-reported PA increased significantly in all intervention groups, utilizing different smartphone apps across 2 months (King et al., 2013) or gain-loss framed text messages for 1 month (Cohen et al., 2017) (studies were evaluated only for pre/post change due to lack of nonmobile control group).

One study achieved PA maintenance in cancer patients undergoing a 1-month intervention with SMS, Fitbit, and telemedicine components (\#18; Gell, Grover, Humble, Sexton, \& Dittus, 2017). Four additional pre/post studies failed to show positive effects based on time in accelerometer-based MVPA (\#35-a 1-month, smartphone app-based intervention focused on interrupting inactivity; Pellegrini et al., 2015), accelerometer-based steps (\#37-a 3-month intervention with education, SMS, and phone probes; Rotheram_Borus at al., 2012), self-reported steps (\#12-a 3-month SMS lifestyle intervention; Donaldson et al., 2014), and selfreport-based MET hours per week (\#15-a 2-week walking intervention with the use of a smartphone pedometer app; Fong et al., 2016). Fong et al. also reported decreases in self-reported steps per day following the 2-week intervention. Additionally, there was no significant change in accelerometer-based steps in a randomized crossover intervention utilizing text messaging, web, and app (\#13; Finkelstein, Bedra, Li, Wood, \& Ouyang, 2015), and a 3-month RCT using an app to target either PA, SB, or both (\#27; Knight et al., 2014). Self-reported participation in $30 \mathrm{~min}$ or more of PA decreased in a 3-month SMS intervention (\#31; McCoy et al., 2017).

Among 29 RCTs with a nonmobile control group, 17 demonstrated improvements in some aspects of PA. The lower part of Figure 2 depicts results of 27 RCTs (with results of one RCT reported across two published papers, \#10 and \#44). Two additional RCTs are mentioned in the text only (\#34, Muntaner-Mas, Vidal-Conti, Borras, Ortega, \& Palou, 2017, and \#36, Pfaeffli Dale et al., 2015). Eight studies depicted in the harvest plot reported increases in self-reported PA indicators (\#2, Antoine-Parker \& Ellis, 2016; \#3, Antypas \& Wangberg, 2014; \#6, Barberan-Garcia et al., 2014; \#7, Block et al., 2015; \#9, Chen et al., 2017; \#10, Chow et al., 2015; \#24, King et al., 2008; and \#44, Thakkar et al., 2016), 
Pre/post studies on sedentary behavior

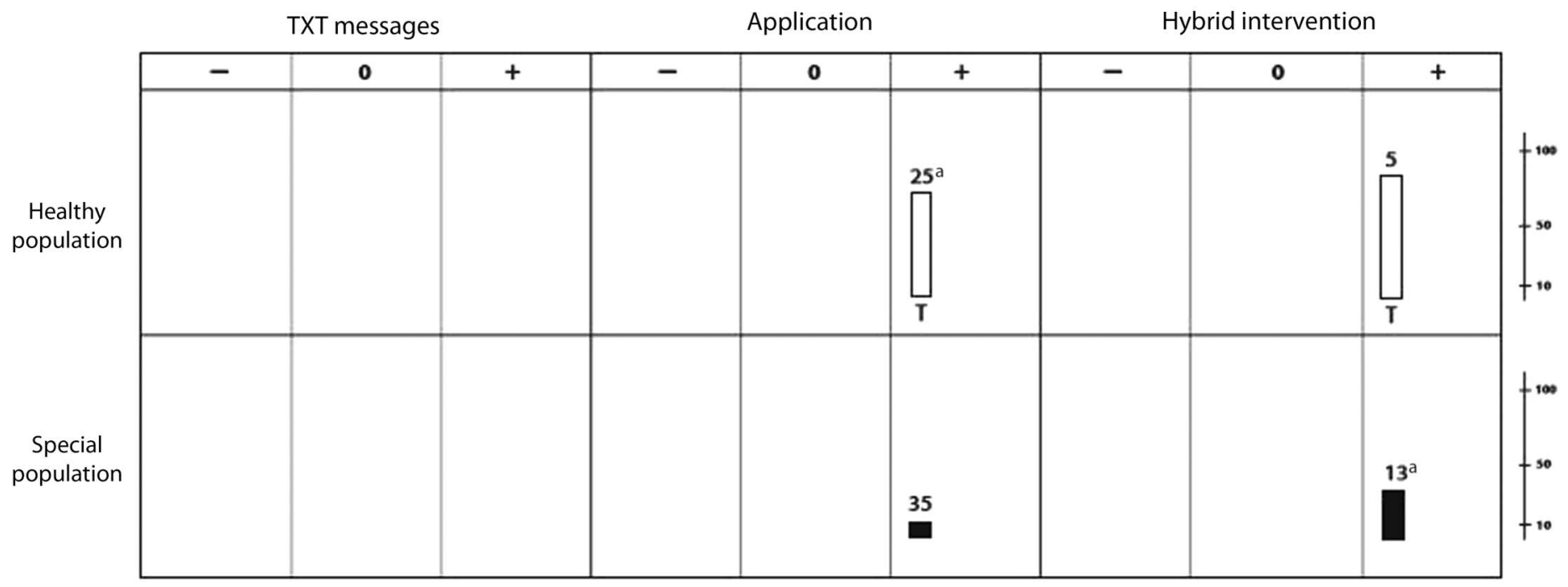

RCT studies on sedentary behavior

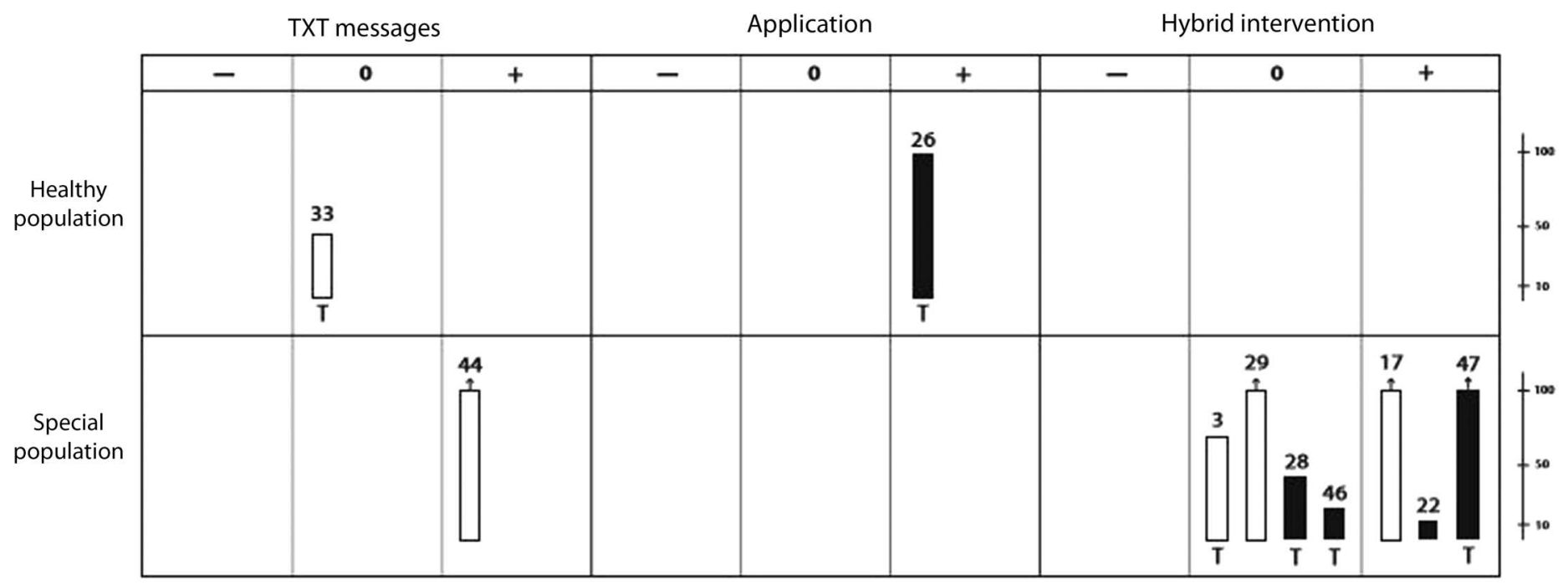

Figure 3 - Harvest plots depicting results of studies targeting sedentary behavior. The bar is placed in the corresponding cell according to the type of intervention (columns), study population (rows), and effect (negative, null, or positive; columns). The size of each bar corresponds to sample size; bars with an arrow pointing upward have $N>100$. Studies with objective outcome measures are indicated with full (black) bars, and studies with subjective outcome measures are indicated with empty (white) bars. The letter "T" below the bar indicates that the intervention was theory based. "RCT treated as pre/ post in harvest plot due to design and nature of comparison. $\mathrm{RCT}=$ randomized control trial.

and seven reported increases in pedometer or accelerometerassessed PA (\#11, Demeyer et al., 2017; \#22, Judice et al., 2015; \#23, Kim \& Glanz, 2013; \#26, King et al., 2016; \#30, Martin et al., 2015; \#47, Thomsen et al., 2017; \#49, van der Weegen et al., 2015). One study reported that assessed MVPA minutes and self-reported vigorous PA were maintained compared with a control group in which it declined (\#14; Fjeldsoe et al., 2016). Improvement in adherence to healthy lifestyle recommendations that included a measure of PA was observed in one study, but data were not reported for PA as an outcome separately (this study is not included in the harvest plot; \#36, Pfaeffli Dale et al., 2015).

Twelve RCT studies failed to demonstrate improvement in PA in comparison with a control group: five based on self-report assessment (IPAQ mainly; \#20, Holmen et al., 2014; \#29, Maddison et al., 2015; \#33, Müller, Khoo, \& Morris, 2016; \#39, Shetty et al., 2011; and \#48, Uhm et al., 2017), four based on pedometer or accelerometer-assessed PA (\#28, Lyons et al., 2017; \#32, Mendelson et al., 2014; \#43, Tabak, VollenbroekHutten, van der Valk, van der Palen, \& Hermens, 2014; and \#45, Thomas et al., 2017), and two studies based on both selfreport and accelerometer data (\#17, Frederix et al., 2015 and \#41, Tabak, Brusse-Keizer, van der Valk, Hermens, \& VollenbroekHutten, 2014). In the case of Frederix et al. (2015), there was a nonsignificant change in accelerometer steps, although the authors also reported improvement in self-reported leisure-time PA (\#17; Frederix et al., 2015). Muntaner-Mas et al. (2017; \#34) reported 
improvements in multiple fitness and health indices following a 10-week Whatsapp-delivered intervention, but the improvements were not statistically different from a control group, and PA data were reported only at baseline (study not included in harvest plot).

Randomized control trial studies with some evidence of effectiveness contained interventions from 1 week to 6 months in length, with six studies including healthy populations and the remaining (23) focusing on clinical populations of older adults. The majority included SMS as a mobile intervention component (but usually as part of a hybrid intervention in combination with selfmonitoring and web support) and reported that theory-guided intervention development (nine studies marked with T in Figure 2, plus a study [\#36] by Pfaeffli Dale et al., 2015). Only three studies that failed to show significant effects reported explicitly that the interventions were guided by theory. Control groups varied and included website access only, self-monitoring only, or usual care, with three studies comparing the use of smartphone apps with different content (\#25 and \#26, King et al., 2013, 2016; and \#27, Knight et al., 2014) and one study comparing gain- versus lossframed messages (\#8; Cohen et al., 2017).

Effect size data for PA were available or could be computed only for 26 of the 50 studies. The mean effect size weighted by sample size was 0.69 , indicating a medium effect size (Cohen's $d=0.86$ and 0.23 , for subjective and objective PA measures, respectively; see Table 2).

Sedentary behavior. Off the 52 studies meeting the inclusion criteria, 14 included measures of SB. Four studies evaluated for pre/post change significantly decreased SB. Two studies had pre/ post single-group design and reported a significant decrease in selfreported sitting time following a 4-month intervention with SMS, Fitbit, and web support (\#5; Ball et al., 2017) and reduced SB (measured by accelerometry) following a 1-month intervention incorporating an app designed to interrupt periods of inactivity (\#35; Pellegrini et al., 2015). Additionally, two randomized studies were treated as pre/post studies in the harvest plots (Figure 3) due to the lack of a control group. Specifically, in a study (\#25) by King et al. (2013), older adults reduced their self-reported television viewing time following a 2-month intervention that utilized three different smartphone apps designed to encourage PA and discourage uninterrupted discretionary sitting time. Similarly, inactivity (assessed with Fitbit as steps not exceeding 20 in $2 \mathrm{hr}$ ) decreased in response to receiving tailored text messages with inactivity reminders for 4 weeks compared with a period with no messages in a randomized crossover study (\#13; Finkelstein et al., 2015).

Among 10 RCTs with a control group, five reported reductions in SB, and five reported no effects of the intervention compared with a control group. In a study (\#26) by King et al. (2016), sedentary time (assessed by built-in smartphone accelerometer) was reduced following the use of a smartphone app focused on social support and interaction compared with an app nurturing affective motives or an analytic app enhancing self-regulation or a diet-tracking control app in a 2-month intervention. Accelerometer-assessed indices of SB also decreased following a 1-week hybrid intervention utilizing SMS, PC alerts to break up sitting, and telephone calls in a study with counterbalanced crossover RCT design (\#22; Judice et al., 2015) and following a 4-month intervention with counseling sessions and SMS reminders to reduce SB (\#47; Thomsen et al., 2017), compared with controls. Self-reported sitting time declined significantly following a 24-week telerehabilitation program that included telemonitoring and tailored text messages once a week to encourage predefined exercise goals (along with messages to improve diet and encourage smoking cessation), compared with a standard center-based cardiac rehab program (\#17; Frederix et al., 2015) and following a 6-month SMS intervention compared with usual care control group (\#44; Thakkar et al., 2016).

Among the five studies that failed to show significant effects were 3- and 4-month hybrid interventions (\#28, Lyons et al., 2017 and \#46, Thomsen et al., 2016) in which SB decreased during the course of the intervention but not significantly compared with a control group. Self-reported SB (assessed as sitting time through IPAQ) did not improve following an intervention with text messaging, web support, and self-monitoring for 6 months (\#29; Maddison et al., 2015), a 3-month intervention with text messaging and online support (\#3; Antypas \& Wangberg, 2014), and a 3-month text messaging intervention compared with an exercise-booklet-only control group (\#33; Müller et al., 2016).

Of the 14 studies with SB data, four studies involved healthy older adults (\#5, Ball et al, 2017; \#25 and \#26, King et al., 2013, 2016; and \#33, Müller et al., 2016), and the remaining studies targeted clinical populations (\#3, Antypas \& Wangberg, 2014; \#13, Finkelstein et al., 2015; \#17, Frederix et al., 2015; \#22, Judice et al., 2015; \#28, Lyons et al., 2017; \#29, Maddison et al., 2015; \#35, Pellegrini et al., 2015; \#44, Thakkar et al., 2016; \#46, Thomsen et al., 2016; and \#47, Thomsen et al., 2017).

Effect size data for SB were available or could be computed for only seven of the 14 studies. The mean effect size weighted by sample size was 0.77, indicating a medium to large effect (Cohen's $d=0.89$ and 0.58 for subjective and objective SB measures, respectively; see Table 2).

Sleep. Five studies reported data on sleep outcomes. Three studies had a single-group pre/post design (\#18, Gell et al., 2017; \#21, Isetta et al., 2017; and \#37 Rotheram-Borus et al., 2012) and two were RCTs (\#7, Block et al., 2015 and \#32, Mendelson et al., 2014). Among the pre/post studies, Gell et al. (\#18) targeted cancer patients in a 1-month intervention with SMS, Fitbit, and telemedicine components and showed improvements in PA but no change in a sleep disturbance score. Rotheram-Borus at al. (\#37) targeted women with diabetes in a 3-month intervention with SMS and on-call buddy groups, which resulted in an increased self-reported sleep duration across the 3-month intervention (with no change in steps). Isetta et al. (\#21) tested a mobile app designed to help patients manage obstructive sleep apnea, including lifestyle recommendations. After 6 weeks of app use, there was no difference in self-reported sleepiness symptoms between those who used the app regularly and irregular users (but regular app users had better CPAP compliance). Among the RCTs, Block et al. (2015) reported significant improvements in sleep (compared with a control group) without providing data or details about sleep measures (\#7; Block et al., 2015). Mendelson et al. (\#32) targeted sleep apnea patients in a 4-month intervention with a mobile app compared with a usual care control group. A self-reported sleepiness score decreased across the intervention, but there was no statistically significant difference between the groups.

Effect size data for sleep were available or could be computed for only two of the four studies. The mean effect size weighted by sample size was 0.46 , indicating a small to medium effect (selfreport outcomes only; see Table 2).

\section{Discussion}

In this paper, we aimed to systematically review intervention approaches involving mobile components (e.g., text messaging, smartphone, or tablet apps) targeting older adults, with their PA, 
$\mathrm{SB}$, and sleep as key behavioral outcomes linked to health and well-being. Our inclusion criteria were fairly liberal in that we sought to identify both quasi-experimental or pre/post single-group studies as well as experimental studies with either self-reported or objectively monitored indicators of the behaviors of interest in adults aged 50 years and older in an effort to evaluate the whole spectrum of existing intervention efforts with mobile technology in older adult populations. This broad approach resulted in the identification of 52 studies with a diverse array of designs, intervention approaches, and subpopulations of older adults.

Overall, it could be stated that mobile interventions have demonstrated some potential to enhance PA and reduce SB in older adults, at least in the short term. With respect to PA, the majority of the studies included in our review provided some evidence of effectiveness for increasing PA (13 out of 21 studies evaluated for pre/post change and 17 out of 29 RCTs). Both self-reported and objectively monitored PA tended to improve with the use of mobile components, with text messaging being the most frequently used component but usually used as part of a hybrid intervention in combination with an app or other online components. Given that cell phone ownership numbers among older adults still significantly exceed numbers for the use of smartphones, text messaging should be an integral component of behavioral interventions targeting older adults. With a few exceptions, most studies were rather short-term (less than 3 months in duration). In some studies that lasted longer, there was evidence that effects tended to subside or be reduced in the long-term (e.g., Barberan-Garcia et al., 2014; Pfaeffli Dale et al., 2015). This, along with evidence that app use may peak as early as within 2 weeks of initiation (Buman et al., 2016) and the fact that one third of consumers abandon their wearables within 6 months (Arthur, 2014), begs the question of what strategies should be used to encourage long-term use or boost usage in specific intervals throughout an intervention. Overall, these conclusions are congruent with the conclusions of the recent review on eHealth PA interventions by Muellmann et al. (2018). In that review, across 20 studies, support was found for short-term (up to 3 months) effectiveness of interventions involving web-, telephone-, or SMS-based interventions. In the present review, which unlike that of Muellmann et al. (2018) focused on mobile interventions and included three types of behaviors (PA, $\mathrm{SB}$, and sleep), only 8 of the studies included long-term follow-up data, with periods of follow-up ranging from 3 (four studies), 6-8 (three studies), to 24 (one study) months. In the 12 studies reviewed by Changizi and Kaveh (2017), the average duration of the interventions was approximately 24 weeks, but no information about long-term follow-up or sustainability of effects was provided.

With respect to $\mathrm{SB}$, nine out of 14 studies indicated that mobile interventions led to reductions in SB. Among RCT studies that assessed self-reported sitting, three failed to show effects, whereas two reduced SB compared with a control group. Among RCT studies with objective measures, all five showed improvement, but in two studies the difference was not statistically significant compared with the control group. Given the fact that all measures come with limitations, it is imperative that researchers incorporate multicomponent measures, combining self-reports with objective data. To the point, although SB is more difficult to recollect accurately, given its automatic, habitual nature, and is thus subject to retrospective and memory biases (Kang \& Rowe, 2015), objective assessment of SB through mobile technology (such as built-in accelerometers) may also result in biased estimates (e.g., inability to differentiate standing still vs. sitting).

It should also be pointed out that many studies targeted both $\mathrm{PA}$ and $\mathrm{SB}$, or at least reported on both outcomes when primarily targeting one behavior (13 out of 14 studies that targeted SB also targeted PA). This is logical and should be encouraged given the complementary nature of the two behaviors and the fact that each independently contributes to health and well-being (Ekelund et al., 2016; Katzmarzyk \& Lee, 2012). The results of the studies reporting both types of outcomes tended to be similar for both types of behaviors, with the exception of three studies presenting significant intervention effects for SB but not for PA (Finkelstein et al., 2015; Frederix et al., 2015; Pellegrini et al., 2015) and one study with effects in the opposite direction (Antypas \& Wangberg, 2014). Since SB may be especially harmful in older adults who fail to meet PA guidelines (Theou, Blodgett, Godin, \& Rockwood, 2017) and PA and SB may also reinforce each other (i.e., sitting displacing activity or the other way around), it is critical that future behavioral interventions targeting older adults include active intervention components that target both behaviors.

We identified only five interventions explicitly focused on improving sleep in older adults through mobile technology. Among these, one study significantly improved self-reported sleep duration through SMS and on-call buddy support (Rotheram-Borus et al., 2012), and Block et al. (2015) reported significant improvements in sleep without specifying outcome measure. Given the fast-advancing field of objective sleep monitoring and commercial mobile apps focused on sleep, we anticipate that these mobile approaches will be applied more widely among older adults in the near future. The trend is already evident from ongoing telemedicine efforts in treating obstructive sleep apnea, in which mobile components are used as complementary tools in supporting mainstream treatments such as CPAP (Isetta et al., 2017; Mendelson et al., 2014). Poor sleep remains a significant health issue for a sizeable proportion of older adults and predicts adverse health outcomes (Friedman, 2016; Grossman, 2017), warranting exploration of how mobile tools could be effectively used to enhance sleep. A good example of intervention efforts utilizing mobile components to such an end is the BeWell24 study by Buman et al. (2016), a smartphone-based intervention targeting sleep, PA, and SB. This study was not included in our review, because the mean age of the sample narrowly missed our predetermined age cutoff (50 years). Nonetheless, the rigorous multistage development process using the Multiphase Optimization Strategy approach (Collins et al., 2007) in this intervention can serve as a good model for developing mobile interventions targeting populations of older adults.

A notable difference between studies with evidence of effectiveness and those that failed to improve (in)activity outcomes concerns the use of theory in guiding intervention development. More studies that reported using theory resulted in improvements compared with those making no mention of theory (especially with PA, see Figure 2), supporting the notion that theoretically driven intervention components may be more effective. Interestingly, Muellmann et al. (2018) reached the same conclusion in their review of eHealth PA interventions for older adults. Whether theory guided intervention development in the studies reviewed by Changizi and Kaveh (2017) was not clear. Additionally, an important step in identifying effective interventions is to design them in a way that allows for evaluation of different intervention components and identification of specific behavior change techniques thought to drive behavior change. Unfortunately, with a few exceptions, most interventions included in this review consisted of multiple components that could not be evaluated separately, and the descriptions of intervention content typically did not involve detailing the specific behavior change techniques used. This, along with rather poor reporting of intervention outcomes, led us to conclude that there 
is much to improve on when it comes to the methodological quality of mHealth studies (an average methodological quality rating of 3.70, ranging from 1 to 5.5 on a 6-point scale). In general, SMS intervention studies tended to have the highest scores, partly due to their study designs, in which effects were more clearly attributable to the SMS component. Admittedly, our assessment of methodological quality was rather crude, and researchers are encouraged to consult original papers when evaluating study details.

Nonetheless, our assessment is consistent with conclusions from recent panels focused on evaluating the potential of digital health intervention efforts. In a recent report, Murray et al. (2016) emphasized the need to establish the likely benefit of a digital health intervention prior to undertaking a costly RCT. An integral part of this process is identifying key intervention components and understanding how they may interact with one another (Murray et al., 2016).

Researchers have pointed out the inherent difficulties in assessing the effectiveness of mHealth interventions through traditional RCT designs, which do not easily allow for continuous adaptation and improvement in response to constantly evolving technologies (Mohr, Cheung, Schueller, Hendricks Brown, \& Duan, 2013). Hence, the evaluation standards for assessing the effectiveness of mHealth interventions may require new paradigms and may need to go beyond traditional study designs (Kumar et al., 2013). Another report from an international panel on digital interventions similarly underscored the need to take advantage of digital approaches in a way that can advance science and theory while at the same time promoting user engagement and evaluating the effectiveness and cost-effectiveness of these intervention approaches (Michie, Yardley, West, Patrick, \& Greaves, 2017). Fortunately, today, researchers can benefit from numerous efforts to create more theoretically sound intervention content (Michie, Ashford, et al., 2011; Michie, van Stralen, \& West, 2011) as well as from approaches for continuous quality improvement and optimization of interventions (Collins, Murphy, \& Strecher, 2007; Collins, NahumShani, \& Almirall, 2014; Kumar et al., 2013; Mohr et al., 2013). With respect to mobile intervention components, an exciting area of intervention development concerns the use of microrandomized controlled designs such as just-in-time adaptive interventions (Moller et al., 2017; Nahum-Shani et al., 2018).

All in all, it was encouraging to see that a wide array of subpopulations of older adults were targeted across the reviewed studies, with representation from both healthy and clinical populations; however, the majority of the studies involved small, nonrepresentative samples. Thus, it is highly likely that the populations represent the more healthy, able, and more technically savvy or tech-inclined older adults (even among the clinical samples). It is imperative that future interventions target underserved populations of older adults, given the evidence that it tends to be the disadvantaged segment of older adults that loses out on the benefits of technology. In a study by Gell et al. (Gell, Rosenberg, Demiris, LaCroix, \& Patel, 2015), for example, technology use varied widely by sociodemographic and health status, such that those who were older had greater physical limitations and greater disability and were less likely to use technology (Internet, e-mail, and text messaging; Gell et al., 2015). Given the disproportionate number of studies targeting healthy versus special populations (27\% healthy vs. $73 \%$ clinical) and the diverse nature of the clinical samples studied, it is difficult to draw conclusions regarding any potential differential effects based on clinical status alone.

Our review was broadly focused in order to evaluate the entire landscape of mHealth approaches targeting older adults with respect to the behavioral outcomes of $\mathrm{PA}, \mathrm{SB}$, and sleep. Nonetheless, the field of mHealth is a fast-developing field, and it is likely that new relevant studies have been published since the completion of this review. In fact, in the course of one year (from the first search we conducted in 2017 to an updated search in January 2018), the number of relevant studies has increased dramatically, underscoring the ever-growing research efforts in this area. Additionally, due to differences in the evaluation and reporting of intervention outcomes, we were unable to depict all studies in the harvest plots. Only about half of the studies reported effect size data or sufficient statistics to compute them. This, along with the diverse approaches to measuring main behavior outcome data, makes it difficult to reliably estimate the overall strength of the effect for the mHealth interventions. In cases in which results were mentioned but data were not reported, we made attempts to contact the authors for supplemental information, but most did not respond to our inquiries. Still of concern is a somewhat piecemeal approach to publishing the results of mHealth intervention studies that occurs when multiple publications report on different outcomes but with data obtained from the same sample. This approach was not always explicitly noted by the authors in the various publications and only became apparent when synthesizing data across studies. It is also possible that we missed some relevant citations. Our search terms focused on mobile devices, smartphones, and text messaging, but studies without these keywords may have been missed. Finally, it must be acknowledged that our search strategy may have led to a bias for positive outcomes, because null outcomes are less likely to be published.

Based on this first review attempting to summarize mHealth intervention efforts targeting behavioral health outcomes in older adults, we can conclude that there is some support for the effectiveness of mHealth approaches for increasing PA and reducing SB in older adults, while research on sleep outcomes is just beginning to accumulate. Still, the generalizability of the effects to the wider older adult population as well as the methodological quality of the existing research could be improved upon. Although progress is being made, the promise of mHealth approaches for enhancing behavioral health in older adults has yet to live up to its expectations. In order for mHealth approaches to be widely embraced by various constituents of aging communities (seniors, health care providers, caregivers, etc.), more research involving high-quality RCTs, new methodological paradigms, and representative samples of older adults is needed.

\section{Acknowledgments}

These results are part of a project that received funding from the European Union's Horizon 2020 research and innovation program under the Marie Skłodowska-Curie, and it is co-financed by the South Moravian Region under grant agreement no. 665860. This material reflects only the authors' attitudes and the European Union is not responsible for any possible use of the information contained in such material. The authors do not have any conflicts of interest. This article does not contain any studies with human participants performed by any of the authors.

\section{References}

Ammenwerth, E., Woess, S., Baumgartner, C., Fetz, B., Van Der Heidt, A., Kastner, P., . . . Poelzl, G. (2015). Evaluation of an integrated telemonitoring surveillance system in patients with coronary heart disease. Methods of Information in Medicine, 54(5), 388-397. doi:10. 3414/ME15-02-0002 
Antoine Parker, C., \& Ellis, R. (2016). Effect of electronic messaging on physical activity participation among older adults. Journal of Aging Research, 2016, 6171028. doi:10.1155/2016/6171028

Antypas, K., \& Wangberg, S.C. (2014). An Internet- and mobile-based tailored intervention to enhance maintenance of physical activity after cardiac rehabilitation: Short-term results of a randomized controlled trial. Journal of Medical Internet Research, 16(3), e77. doi:10.2196/ jmir.3132

Arem, H., Moore, S.C., Patel, A., Hartge, P., Berrington de Gonzalez, A., Visvanathan, K., . . . Matthews, C.E. (2015). Leisure time physical activity and mortality. JAMA Internal Medicine, 175(6), 959. PubMed ID: 25844730 doi:10.1001/jamainternmed.2015.0533

Arsand, E., Tatara, N., Ostengen, G., \& Hartvigsen, G. (2010). Mobile phone-based self-management tools for type 2 diabetes: The few touch application. Journal of Diabetes Science and Technology, 4(2), 328-336. PubMed ID: 20307393 doi:10.1177/193229681000400213

Arthur, C. (2014). Wearables: One-third of consumers abandoning devices. The Guardian. Retrieved from https://www.theguardian.com/ technology/2014/apr/01/wearables-consumers-abandoning-devicesgalaxy-gear

Ball, K., Hunter, R.F., Maple, J.-L., Moodie, M., Salmon, J., Ong, K.-L., ... Crawford, D. (2017). Can an incentive-based intervention increase physical activity and reduce sitting among adults? The ACHIEVE (Active Choices IncEntiVE) feasibility study. International Journal of Behavioral Nutrition and Physical Activity, 14(1), 35. PubMed ID: 28320409 doi:10.1186/s12966-017-0490-2

Barberan-Garcia, A., Vogiatzis, I., Solberg, H.S., Vilaró, J., Rodríguez, D.A., Garåsen, H.M., . . . NEXES Consortium. (2014). Effects and barriers to deployment of telehealth wellness programs for chronic patients across 3 European countries. Respiratory Medicine, 108(4), 628-637. PubMed ID: 24451438 doi:10.1016/j.rmed.2013.12.006

Beishuizen, C.R., Stephan, B.C., van Gool, W.A., Brayne, C., Peters, R.J., Andrieu, S., . . . Richard, E. (2016). Web-based interventions targeting cardiovascular risk factors in middle-aged and older people: A systematic review and meta-analysis. Journal of Medical Internet Research, 18(3), e55. PubMed ID: 26968879 doi:10.2196/ jmir.5218

Block, G., Azar, K.M.J., Romanelli, R.J., Block, T.J., Hopkins, D., Carpenter, H.A., . . . Block, C.H. (2015). Diabetes prevention and weight loss with a fully automated behavioral intervention by email, Web, and mobile phone: A randomized controlled trial among persons with prediabetes. Journal of Medical Internet Research, 17(10), e240. PubMed ID: 26499966 doi:10.2196/jmir.4897

Buman, M.P., Epstein, D.R., Gutierrez, M., Herb, C., Hollingshead, K., Huberty, J.L., . . Baldwin, C.M. (2016). BeWell24: development and process evaluation of a smartphone "app" to improve sleep, sedentary, and active behaviors in US Veterans with increased metabolic risk. Translational Behavioral Medicine, 6(3), 438-448. PubMed ID: 27528532 doi:10.1007/s13142-015-0359-3

Buman, M.P., Winkler, E.A.H., Kurka, J.M., Hekler, E.B., Baldwin, C.M., Owen, N., . . Gardiner, P.A. (2014). Reallocating time to sleep, sedentary behaviors, or active behaviors: Associations with cardiovascular disease risk biomarkers, NHANES 2005-2006. American Journal of Epidemiology, 179(3), 323-334. PubMed ID: 24318278 doi:10.1093/aje/kwt292

Changizi, M., \& Kaveh, M.H. (2017). Effectiveness of the mHealth technology in improvement of healthy behaviors in an elderly population-A systematic review. mHealth, 3, 51. PubMed ID: 29430455 doi:10.21037/mhealth.2017.08.06

Chen, H.C., Chuang, T.Y., Lin, P.C., Lin, Y.K., \& Chuang, Y.H. (2017). Effects of messages delivered by mobile phone on increasing compliance with shoulder exercises among patients with a frozen shoulder. Journal of Nursing Scholarship, 49(4), 429-437. PubMed ID: 28632975 doi:10.1111/jnu.12308

Chiarini, G., Ray, P., Akter, S., Masella, C., \& Ganz, A. (2013). mHealth technologies for chronic diseases and elders: A systematic review. IEEE Journal on Selected Areas in Communications, 31(9), 6-18. doi:10.1109/JSAC.2013.SUP.0513001

Chow, C.K., Redfern, J., Hillis, G.S., Thakkar, J., Santo, K., Hackett, M.L., . . . Thiagalingam, A. (2015). Effect of lifestyle-focused text messaging on risk factor modification in patients with coronary heart disease. JAMA, 314(12), 1255. PubMed ID: 26393848 doi:10.1001/ jama.2015.10945

Cohen, A., Perozich, A., Rajan, R., Persky, S., Parisi, J., Bowie, J., . . . Cheskin, L.J. (2017). Framed, interactive theory-driven texting: Effects of message framing on health behavior change for weight loss. Family \& Community Health, 40(1), 43-51. PubMed ID: 27870754 doi:10.1097/FCH.0000000000000128

Collins, L.M., Murphy, S.A., \& Strecher, V. (2007). The Multiphase Optimization Strategy (MOST) and the Sequential Multiple Assignment Randomized Trial (SMART): New methods for more potent eHealth interventions. American Journal of Preventive Medicine, 32(5 Suppl.), S112-S118. doi:10.1016/j.amepre.2007.01.022

Collins, L.M., Nahum-Shani, I., \& Almirall, D. (2014). Optimization of behavioral dynamic treatment regimens based on the Sequential, Multiple Assignment, Randomized Trial (SMART). Clinical Trials, 11(4), 426-434. PubMed ID: 24902922 doi:10.1177/ 1740774514536795

Demeyer, H., Louvaris, Z., Frei, A., Rabinovich, R.A., de Jong, C., GimenoSantos, E., . . . Mr Papp PROactive study group and the PROactive consortium. (2017). Physical activity is increased by a 12-week semiautomated telecoaching programme in patients with COPD: A multicentre randomised controlled trial. Thorax, 72(5), 415-423. PubMed ID: 28137918 doi:10.1136/thoraxjnl-2016-209026

Donaldson, E.L., Fallows, S., \& Morris, M. (2014). A text message based weight management intervention for overweight adults. Journal of Human Nutrition and Dietetics, 27(Suppl. 2), 90-97. doi:10.1111/ jhn.12096

Drewnowski, A., \& Evans, W.J. (2001). Nutrition, physical activity, and quality of life in older adults: Summary. The Journals of Gerontology, Series A: Biological Sciences \& Medical Sciences, 56(Spec. No. 2), 89-94. doi:10.1093/gerona/56.suppl_2.89

Ekelund, U., Steene-Johannessen, J., Brown, W.J., Fagerland, M.W., Owen, N., Powell, K.E., . . . Lancet Sedentary Behaviour Working Group. (2016). Does physical activity attenuate, or even eliminate, the detrimental association of sitting time with mortality? A harmonised metaanalysis of data from more than 1 million men and women. The Lancet, 388(10051), 1302-1310. doi:10.1016/S0140-6736(16)30370-1

Eurostat. (2016). Internet access and use statistics-Households and individuals-Statistics Explained. Retrieved from https://ec.europa. eu/eurostat/statistics-explained/index.php?title=Archive:Internet_ access_and_use_statistics_-_households_and_individuals

Finkelstein, J., Bedra, M., Li, X., Wood, J., \& Ouyang, P. (2015). Mobile app to reduce inactivity in sedentary overweight women. Studies in Health Technology and Informatics, 216, 89-92. PubMed ID: 26262016 doi:10.3233/978-1-61499-564-7-89

Fjeldsoe, B.S., Goode, A.D., Phongsavan, P., Bauman, A., Maher, G., Winkler, E., \& Eakin, E.G. (2016). Evaluating the maintenance of lifestyle changes in a randomized controlled trial of the 'Get Healthy, Stay Healthy' program. JMIR mHealth and uHealth, 4(2), e42. doi: $10.2196 /$ mhealth. 5280

Fong, S.S., Ng, S.S., Cheng, Y.T., Zhang, J., Chung, L.M., Chow, G.C., . . . Macfarlane, I.M. (2016). Comparison between smartphone pedometer applications and traditional pedometers for improving 
physical activity and body mass index in community-dwelling older adults. Journal of Physical Therapy Science, 28(5), 1651-1656. doi:10.1589/jpts.28.1651

Forman, D.E., LaFond, K., Panch, T., Allsup, K., Manning, K., \& Sattelmair, J. (2014). Utility and efficacy of a smartphone application to enhance the learning and behavior goals of traditional cardiac rehabilitation: A feasibility study. Journal of Cardiopulmonary Rehabilitation and Prevention, 34(5), 327-334. PubMed ID: 24866355 doi:10.1097/HCR.0000000000000058

Frederix, I., Hansen, D., Coninx, K., Vandervoort, P., Vandijck, D., Hens, N., . . Dendale, P. (2015). Medium-term effectiveness of a comprehensive Internet-based and patient-specific telerehabilitation program with text messaging support for cardiac patients: Randomized controlled trial. Journal of Medical Internet Research, 17(7), e185. PubMed ID: 26206311 doi:10.2196/jmir.4799

Friedman, E.M. (2016). Self-reported sleep problems prospectively increase risk of disability: Findings from the survey of midlife development in the United States. Journal of the American Geriatrics Society, 64(11), 2235-2241. PubMed ID: 27626617 doi:10.1111/jgs. 14347

Gell, N.M., Grover, K.W., Humble, M., Sexton, M., \& Dittus, K. (2017). Efficacy, feasibility, and acceptability of a novel technology-based intervention to support physical activity in cancer survivors. Supportive Care in Cancer, 25(4), 1291-1300. PubMed ID: 27957621 doi:10.1007/s00520-016-3523-5

Gell, N.M., Rosenberg, D.E., Demiris, G., LaCroix, A.Z., \& Patel, K.V. (2015). Patterns of technology use among older adults with and without disabilities. The Gerontologist, 55(3), 412-421. PubMed ID: 24379019 doi:10.1093/geront/gnt166

Geraedts, H., Zijlstra, A., Bulstra, S.K., Stevens, M., \& Zijlstra, W. (2013). Effects of remote feedback in home-based physical activity interventions for older adults: A systematic review. Patient Education and Counseling, 91(1), 14-24. PubMed ID: 23194823 doi:10.1016/j.pec. 2012.10.018

Grossman, E.S. (2017). Enduring sleep complaints predict health problems: A six-year follow-up of the survey of health and retirement in Europe. Aging \& Mental Health, 21(11), 1155-1163. PubMed ID: 27484858 doi:10.1080/13607863.2016.1209735

Haeger, M., Bock, O., \& Zijlstra, W. (2017). A smartphone based approach to enhance older persons' mobility in daily life. Gerontechnology, 16(2), 109-114. doi:10.4017/gt.2017.16.2.006.00

Helbostad, J.L., Vereijken, B., Becker, C., Todd, C., Taraldsen, K., Pijnappels, M., . . . Mellone, S. (2017). Mobile health applications to promote active and healthy ageing. Sensors, 17(3), E622. doi:10. 3390/s17030622

Holmen, H., Torbjørnsen, A., Wahl, A.K., Jenum, A.K., Småstuen, M.C., Årsand, E., \& Ribu, L. (2014). A mobile health intervention for selfmanagement and lifestyle change for persons with type 2 diabetes, part 2: One-year results from the Norwegian randomized controlled trial renewing health. JMIR mHealth and uHealth, 2(4), e57. PubMed ID: 25499872 doi:10.2196/mhealth.3882

Isetta, V., Torres, M., González, K., Ruiz, C., Dalmases, M., Embid, C., . . Montserrat, J.M. (2017). A new mHealth application to support treatment of sleep apnoea patients. Journal of Telemedicine and Telecare, 23(1), 14-18. PubMed ID: 26672606 doi:10.1177/ $1357633 X 15621848$

Júdice, P.B., Hamilton, M.T., Sardinha, L.B., \& Silva, A.M. (2015). Randomized controlled pilot of an intervention to reduce and break-up overweight/obese adults' overall sitting-time. Trials, 16(1), 490. doi:10.1186/s13063-015-1015-4

Kampmeijer, R., Pavlova, M., Tambor, M., Golinowska, S., \& Groot, W. (2016). The use of e-health and m-health tools in health promotion and primary prevention among older adults: A systematic literature review. BMC Health Services Research, 16(S5), 290. doi:10.1186/ s12913-016-1522-3

Kang, M., \& Rowe, D.A. (2015). Issues and challenges in sedentary behavior measurement. Measurement in Physical Education and Exercise Science, 19(3), 105-115. doi:10.1080/1091367X.2015. 1055566

Katzmarzyk, P.T., \& Lee, I.-M. (2012). Sedentary behaviour and life expectancy in the USA: A cause-deleted life table analysis. $B M J$ Open, 2(4), e000828. PubMed ID: 22777603 doi:10.1136/bmjopen2012-000828

Kim, B.Y., \& Glanz, K. (2013). Text messaging to motivate walking in older African Americans: A randomized controlled trial. American Journal of Preventive Medicine, 44(1), 71-75. doi:10.1016/j.amepre. 2012.09.050

Kim, B.Y., \& Lee, J. (2017). Smart devices for older adults managing chronic disease: A scoping review. JMIR mHealth and uHealth, 5(5), e69. PubMed ID: 28536089 doi:10.2196/mhealth.7141

King, A.C., Ahn, D.K., Oliveira, B.M., Atienza, A.A., Castro, C.M., \& Gardner, C.D. (2008). Promoting physical activity through hand-held computer technology. American Journal of Preventive Medicine, 34(2), 138-142. PubMed ID: 18201644 doi:10.1016/j.amepre. 2007.09.025

King, A.C., Hekler, E.B., Grieco, L.A., Winter, S.J., Sheats, J.L., Buman, M.P., . . . Cirimele, J. (2013). Harnessing different motivational frames via mobile phones to promote daily physical activity and reduce sedentary behavior in aging adults. PLoS ONE, 8(4), e62613. PubMed ID: 23638127 doi:10.1371/journal.pone.0062613

King, A.C., Hekler, E.B., Grieco, L.A., Winter, S.J., Sheats, J.L., Buman, M.P., . . Cirimele, J. (2016). Effects of three motivationally targeted mobile device applications on initial physical activity and sedentary behavior change in midlife and older adults: A randomized trial. PLoS ONE, 11(6), e0156370. PubMed ID: 27352250 doi:10.1371/journal. pone. 0156370

Knight, E., Stuckey, M.I., \& Petrella, R.J. (2014). Health promotion through primary care: Enhancing self-management with activity prescription and mHealth. The Physician and Sportsmedicine, 42(3), 90-99. doi:10.3810/psm.2014.09.2080

Knowles, L.M., Skeath, P., Jia, M., Najafi, B., Thayer, J., \& Sternberg, E.M. (2016). New and future directions in integrative medicine research methods with a focus on aging populations: A review. Gerontology, 62(4), 467-476. PubMed ID: 26545038 doi:10.1159/ 000441494

Koster, A., Penninx, B.W., Newman, A.B., Visser, M., van Gool, C.H., Harris, T.B., . . . Kritchevsky, S.B. (2007). Lifestyle factors and incident mobility limitation in obese and non-obese older adults. Obesity, 15(12), 3122-3132. doi:10.1038/oby.2007.372

Kruse, C.S., Mileski, M., \& Moreno, J. (2017). Mobile health solutions for the aging population: A systematic narrative analysis. Journal of Telemedicine and Telecare, 23(4), 439-451. doi:10.1177/ $1357633 X 16649790$

Kumar, S., Nilsen, W.J., Abernethy, A., Atienza, A., Patrick, K., Pavel, M., . . Swendeman, D. (2013). Mobile health technology evaluation. American Journal of Preventive Medicine, 45(2), 228-236. PubMed ID: 23867031 doi:10.1016/j.amepre.2013.03.017

Lyons, E.J., Swartz, M.C., Lewis, Z.H., Martinez, E., \& Jennings, K. (2017). Feasibility and acceptability of a wearable technology physical activity intervention with telephone counseling for mid-aged and older adults: A randomized controlled pilot trial. JMIR mHealth and uHealth, 5(3), e28. doi:10.2196/mhealth.6967

Maddison, R., Pfaeffli, L., Whittaker, R., Stewart, R., Kerr, A., Jiang, Y., . . Rawstorn, J. (2015). A mobile phone intervention increases 
physical activity in people with cardiovascular disease: Results from the HEART randomized controlled trial. European Journal of Preventive Cardiology, 22(6), 701-709. PubMed ID: 24817694 doi:10. 1177/2047487314535076

Martin, S.S., Feldman, D.I., Blumenthal, R.S., Jones, S.R., Post, W.S., McKibben, R.A., . . . Blaha, M.J. (2015). mActive: A randomized clinical trial of an automated mHealth intervention for physical activity promotion. Journal of the American Heart Association, 4(11), e002239. PubMed ID: 26553211 doi:10.1161/JAHA.115. 002239

McCoy, P., Leggett, S., Bhuiyan, A., Brown, D., Frye, P., \& Williams, B. (2017). Text messaging: An intervention to increase physical activity among African American participants in a faith-based, competitive weight loss program. International Journal of Environmental Research and Public Health, 14(4), 326. doi:10.3390/ ijerph14040326

Mekary, R.A., Willett, W.C., Hu, F.B., \& Ding, E.L. (2009). Isotemporal substitution paradigm for physical activity epidemiology and weight change. American Journal of Epidemiology, 170(4), 519-527. PubMed ID: 19584129 doi:10.1093/aje/kwp163

Mendelson, M., Vivodtzev, I., Tamisier, R., Laplaud, D., Dias-Domingos, S., Baguet, J.-P., . . Pépin, J.-L. (2014). CPAP treatment supported by telemedicine does not improve blood pressure in high cardiovascular risk OSA patients: A randomized, controlled trial. Sleep, 37(11), 1863-1870. PubMed ID: 25364081 doi:10.5665/sleep.4186

Michie, S., Ashford, S., Sniehotta, F.F., Dombrowski, S.U., Bishop, A., \& French, D.P. (2011). A refined taxonomy of behaviour change techniques to help people change their physical activity and healthy eating behaviours: The CALO-RE taxonomy. Psychology \& Health, 26(11), 1479-1498. PubMed ID: 21678185 doi:10.1080/08870446. 2010.540664

Michie, S., van Stralen, M.M., \& West, R. (2011). The behaviour change wheel: a new method for characterising and designing behaviour change interventions. Implementation Science, 6, 42. PubMed ID: 21513547 doi:10.1186/1748-5908-6-42

Michie, S., Yardley, L., West, R., Patrick, K., \& Greaves, F. (2017). Developing and evaluating digital interventions to promote behavior change in health and health care: Recommendations resulting from an international workshop. Journal of Medical Internet Research, 19(6), e232. PubMed ID: 28663162 doi:10.2196/jmir.7126

Moher, D., Liberati, A., Tetzlaff, J., Altman, D.G., \& PRISMA Group. (2009). Preferred reporting items for systematic reviews and metaanalyses: The PRISMA statement. PLoS Medicine, 6(7), e1000097. PubMed ID: 19621072 doi:10.1371/journal.pmed.1000097

Mohr, D.C., Cheung, K., Schueller, S.M., Hendricks Brown, C., \& Duan, N. (2013). Continuous evaluation of evolving behavioral intervention technologies. American Journal of Preventive Medicine, 45(4), 517523. PubMed ID: 24050429 doi:10.1016/j.amepre.2013.06.006

Moller, A.C., Merchant, G., Conroy, D.E., West, R., Hekler, E., Kugler, K.C., \& Michie, S. (2017). Applying and advancing behavior change theories and techniques in the context of a digital health revolution: Proposals for more effectively realizing untapped potential. Journal of Behavioral Medicine, 40(1), 85-98. PubMed ID: 28058516 doi:10. 1007/s10865-016-9818-7

Moore, S.C., Patel, A.V., Matthews, C.E., Berrington de Gonzalez, A., Park, Y., Katki, H., . . . Lee, I.M. (2012). Leisure time physical activity of moderate to vigorous intensity and mortality: A large pooled cohort analysis. PLoS Medicine, 9(11), e1001335. PubMed ID: 23139642 doi:10.1371/journal.pmed.1001335

Muellmann, S., Forberger, S., Möllers, T., Bröring, E., Zeeb, H., \& Pischke, C.R. (2018). Effectiveness of eHealth interventions for the promotion of physical activity in older adults: A systematic review. Preventive Medicine, 108, 93-110. PubMed ID: 29289643 doi:10.1016/j.ypmed.2017.12.026

Müller, A.M., Khoo, S., \& Morris, T. (2016). Text messaging for exercise promotion in older adults from an upper-middle-income country: Randomized controlled trial. Journal of Medical Internet Research, 18(1), e5. doi:10.2196/jmir.5235

Muntaner-Mas, A., Vidal-Conti, J., Borras, P.A., Ortega, F.B., \& Palou, P. (2017). Effects of a Whatsapp-delivered physical activity intervention to enhance health-related physical fitness components and cardiovascular disease risk factors in older adults. The Journal of Sports Medicine and Physical Fitness, 57(1-2), 91-102. PubMed ID: 28604654

Murray, E., Hekler, E.B., Andersson, G., Collins, L.M., Doherty, A., Hollis, C., . . . Wyatt, J.C. (2016). Evaluating digital health interventions: Key questions and approaches. American Journal of Preventive Medicine, 51(5), 843-851. PubMed ID: 27745684 doi:10. 1016/j.amepre.2016.06.008

Nahum-Shani, I., Smith, S.N., Spring, B.J., Collins, L.M., Witkiewitz, K., Tewari, A., \& Murphy, S.A. (2018). Just-in-Time Adaptive Interventions (JITAIs) in mobile health: Key components and design principles for ongoing health behavior support. Annals of Behavioral Medicine, 52(6), 446-462. doi:10.1007/s12160-016-9830-8

Ogilvie, D., Fayter, D., Petticrew, M., Sowden, A., Thomas, S., Whitehead, M., \& Worthy, G. (2008). The harvest plot: A method for synthesising evidence about the differential effects of interventions. BMC Medical Research Methodology, 8(1), 8. doi:10.1186/ 1471-2288-8-8

Peel, N.M., McClure, R.J., \& Bartlett, H.P. (2005). Behavioral determinants of healthy aging. American Journal of Preventive Medicine, 28(3), 298-304. PubMed ID: 15766620 doi:10.1016/j.amepre.2004. 12.002

Pellegrini, C.A., Hoffman, S.A., Daly, E.R., Murillo, M., Iakovlev, G., \& Spring, B. (2015). Acceptability of smartphone technology to interrupt sedentary time in adults with diabetes. Translational Behavioral Medicine, 5(3), 307-314. PubMed ID: 26327936 doi:10.1007/ s13142-015-0314-3

PEW Research Center. (2014). Older adults and technology use. Retrieved from http://www.pewinternet.org/2014/04/03/older-adults-andtechnology-use/\%0A

PEW Research Center. (2016). Smartphone ownership and Internet usage continues to climb in emerging economies Pew Research Center. Retrieved from http://www.pewglobal.org/2016/02/22/smartphoneownership-and-internet-usage-continues-to-climb-in-emergingeconomies/

PEW Research Center. (2017). Mobile fact sheet. Retrieved from http:// www.pewinternet.org/fact-sheet/mobile/

Pfaeffli Dale, L., Whittaker, R., Jiang, Y., Stewart, R., Rolleston, A., \& Maddison, R. (2015). Text message and Internet support for coronary heart disease self-management: Results from the Text4Heart randomized controlled trial. Journal of Medical Internet Research, 17(10), e237. PubMed ID: 26490012 doi:10.2196/ jmir.4944

Rotheram-Borus, M.J., Tomlinson, M., Gwegwe, M., Comulada, W.S., Kaufman, N., \& Keim, M. (2012). Diabetes buddies: Peer support through a mobile phone buddy system. The Diabetes Educator, 38(3), 357-365. PubMed ID: 22546740 doi:10.1177/0145721712444617

Shetty, A.S., Chamukuttan, S., Nanditha, A., Raj, R.K., \& Ramachandran, A. (2011). Reinforcement of adherence to prescription recommendations in Asian Indian diabetes patients using short message service (SMS) - A pilot study. Journal of Association of Physicians of India, 59(11), 711-714. Retrieved from http://www.japi.org/ november2011/04_oa.pdf 
Silveira, P., van de Langenberg, R., van het Reve, E., Daniel, F., Casati, F., $\&$ de Bruin, E.D. (2013). Tablet-based strength-balance training to motivate and improve adherence to exercise in independently living older people: A phase II preclinical exploratory trial. Journal of Medical Internet Research, 15(8), e159. PubMed ID: 23939401 doi:10.2196/jmir.2579

Stuckey, M., Russell-Minda, E., Read, E., Munoz, C., Shoemaker, K., Kleinstiver, P., \& Petrella, R. (2011). Diabetes and Technology for Increased Activity (DaTA) study: Results of a remote monitoring intervention for prevention of metabolic syndrome. Journal of Diabetes Science and Technology, 5(4), 928-935. PubMed ID: 21880236 doi:10.1177/193229681100500416

Tabak, M., Brusse-Keizer, M., van der Valk, P., Hermens, H., \& Vollenbroek-Hutten, M. (2014). A telehealth program for selfmanagement of COPD exacerbations and promotion of an active lifestyle: A pilot randomized controlled trial. International Journal of Chronic Obstructive Pulmonary Disease, 9, 935-944. PubMed ID: 25246781 doi:10.2147/COPD.S60179

Tabak, M., op den Akker, H., \& Hermens, H. (2014). Motivational cues as real-time feedback for changing daily activity behavior of patients with COPD. Patient Education and Counseling, 94(3), 372-378. PubMed ID: 24332934 doi:10.1016/j.pec.2013.10.014

Tabak, M., Vollenbroek-Hutten, M.M., van der Valk, P.D., van der Palen, J., \& Hermens, H.J. (2014). A telerehabilitation intervention for patients with chronic obstructive pulmonary disease: A randomized controlled pilot trial. Clinical Rehabilitation, 28(6), 582-591. PubMed ID: 24293120 doi:10.1177/0269215513512495

Taraldsen, K., Chastin, S.F., Riphagen, I.I., Vereijken, B., \& Helbostad, J.L. (2012). Physical activity monitoring by use of accelerometerbased body-worn sensors in older adults: A systematic literature review of current knowledge and applications. Maturitas, 71(1), 1319. PubMed ID: 22134002 doi:10.1016/j.maturitas.2011.11.003

Thakkar, J., Redfern, J., Thiagalingam, A., \& Chow, C.K. (2016). Patterns, predictors and effects of texting intervention on physical activity in CHD_Insights from the TEXT ME randomized clinical trial. European Journal of Preventive Cardiology, 23(17), 1894-1902. PubMed ID: 27512051 doi:10.1177/2047487316664190

Theou, O., Blodgett, J.M., Godin, J., \& Rockwood, K. (2017). Association between sedentary time and mortality across levels of frailty. CMAJ: Canadian Medical Association Journal, 189(33), E1056-E1064. doi:10.1503/cmaj.161034

Thomas, J.G., Raynor, H.A., Bond, D.S., Luke, A.K., Cardoso, C.C., Foster, G.D., \& Wing, R.R. (2017). Weight loss in Weight Watchers Online with and without an activity tracking device compared to control: A randomized trial. Obesity, 25(6), 1014-1021. PubMed ID: 28437597 doi:10.1002/oby. 21846
Thomsen, T., Aadahl, M., Beyer, N., Hetland, M.L., Løppenthin, K., Midtgaard, J., . . . Esbensen, B.A. (2016). Motivational counselling and SMS-reminders for reduction of daily sitting time in patients with rheumatoid arthritis: A descriptive randomised controlled feasibility study. BMC Musculoskeletal Disorders, 17(1), 434. PubMed ID: 27756265 doi:10.1186/s12891-016-1266-6

Thomsen, T., Aadahl, M., Beyer, N., Hetland, M.L., Løppenthin, K., Midtgaard, J., . . . Esbensen, B.A. (2017). The efficacy of motivational counselling and SMS reminders on daily sitting time in patients with rheumatoid arthritis: A randomised controlled trial. Annals of the Rheumatic Diseases, 76(9), 1603-1606. doi:10.1136/annrheumdis2016-210953

Uhm, K.E., Yoo, J.S., Chung, S.H., Lee, J.D., Lee, I., Kim, J.I., . . . Hwang, J.H. (2017). Effects of exercise intervention in breast cancer patients: is mobile health (mHealth) with pedometer more effective than conventional program using brochure? Breast Cancer Research and Treatment, 161(3), 443-452. PubMed ID: 27933450 doi:10. 1007/s10549-016-4065-8

Van der Weegen, S., Verwey, R., Spreeuwenberg, M., Tange, H., van der Weijden, T., \& de Witte, L. (2015). It's life! Mobile and web-based monitoring and feedback tool embedded in primary care increases physical activity: A cluster randomized controlled trial. Journal of Medical Internet Research, 17(7), e184. PubMed ID: 26209025 doi:10.2196/jmir.4579

Vathsangam, H., \& Sukhatme, G.S. (2014). Using phone-based activity monitors to promote physical activity in older adults: A pilot study. Presented at the2014 IEEE Healthcare Innovation Conference (HIC), Seattle, WA (pp. 42-47). doi:10.1109/HIC.2014.7038870

Verwey, R., van der Weegen, S., Spreeuwenberg, M., Tange, H., van der Weijden, T., \& de Witte, L. (2014). A pilot study of a tool to stimulate physical activity in patients with COPD or type 2 diabetes in primary care. Journal of Telemedicine and Telecare, 20(1), 29-34. PubMed ID: 24414397 doi:10.1177/1357633X13519057

World Health Organization. (2016a). WHO international classification of health interventions (ICHI). Retrieved from http://www.who.int/ classifications/ichi/en/

World Health Organization. (2016b). WHO monitoring and evaluating digital health interventions. Retrieved from http://www.who.int/ reproductivehealth/publications/mhealth/digital-health-interventions/ en/

Zolfaghari, M., Mousavifar, S.A., Pedram, S., \& Haghani, H. (2012). Retracted: The impact of nurse short message services and telephone follow-ups on diabetic adherence: Which one is more effective? Journal of Clinical Nursing, 21(13-14), 1922-1931. doi:10.1111/j.1365-2702. 2011.03951.x 